\title{
1 NK cell immune responses differ after prime and boost vaccination
}

2 Jean-Louis Palgen ${ }^{1,2}$, Nicolas Tchitchek ${ }^{1,2}$, Nicolas Huot ${ }^{2,3}$, Jamila Elhmouzi-Younes ${ }^{1,2}$, Cécile

3 Lefebvre $^{2,4}$, Pierre Rosenbaum ${ }^{1,2}$, Nathalie Dereuddre-Bosquet ${ }^{1,2}$, Frédéric Martinon ${ }^{1,2}$, Hakim

4 Hocini $^{2,4}$, Antonio Cosma ${ }^{1,2}$, Michaela Müller-Trutwin ${ }^{2,3}$, Yves Lévy ${ }^{2,4}$, Roger Le Grand ${ }^{1,2}$, and

5 Anne-Sophie Beignon ${ }^{1,2, *}$

6

$7{ }^{1}$ CEA - Université Paris Sud 11 - INSERM U1184, Immunology of Viral Infections and 8 Autoimmune Diseases, IDMIT department, IBFJ, 92265 Fontenay-aux-Roses, France

$9 \quad{ }^{2}$ Vaccine Research Institute, Henri Mondor Hospital, 94010 Créteil, France

$10{ }^{3}$ Institut Pasteur, Unit on HIV, Inflammation and Persistance, 75015 Paris, France

$11{ }^{4}$ Institut Mondor de Recherche Biomédicale - INSERM U955, Eq.16, 94010, Créteil, France

12 *Corresponding author: Anne-Sophie Beignon; 18, route du Panorama; 92265 Fontenay-aux-

13 Roses, France; Phone: +33 1465480 27; Fax: +33 1465477 26; email: anne-

14 sophie.beignon@cea.fr

Short title: NK cell response after immunizations 
Abbreviations:

21 CBC: complete blood count

22 DC: dendritic cell

23 HIV: human immunodeficiency virus

24 LASSO: Least Absolute Shrinkage and Selection Operator

25 LDA: Linear Discriminant Analysis

26 MCMV: mouse cytomegalovirus

$27 \mathrm{MSI}$ : mean signal intensity

28 MVA: modified vaccinia virus Ankara

29 NHP: nonhuman primates

$30 \quad$ NK: natural killer

31 PBMC: peripheral blood mononuclear cell

32 SPADE: Spanning-tree Progression Analyses of Density-normalized Events

33 
A better understanding of innate responses induced by vaccination is critical for designing optimal vaccines. Here, we studied the diversity and dynamics of the NK cell compartment after prime-boost immunization with the modified vaccinia virus Ankara using cynomolgus macaques as a model. Mass cytometry was used to deeply characterize blood NK cells. The NK cell subphenotype composition was modified by the prime. Certain phenotypic changes induced by the prime were maintained over time and, as a result, the NK cell composition prior to boost differed from that before prime. The key phenotypic signature that distinguished NK cells responding to the boost from those responding to the prime included stronger expression of several cytotoxic, homing, and adhesion molecules, suggesting that NK cells at recall were functionally distinct. Our data reveal potential priming or imprinting of NK cells after the first vaccine injection. This study provides novel insights into prime-boost vaccination protocols that could be used to optimize future vaccines.

47

Keywords: innate lymphoid immunity, NK cells, prime-boost, vaccination, MVA, NHP, mass 49 
A better understanding of the early events following vaccination is critical for identifying key biomarkers and mechanisms involved in the subsequent establishment of immune memory to optimize future vaccines (1). This requires extensive characterization of the vaccineinduced innate immune response.

Natural Killer (NK) cells are innate lymphoid cells that can constitutively kill cells carrying an abnormal MHC signature, via interactions of activating and inhibitory receptors between NK cells and their targets (2-4). The wide diversity of the NK cell receptor repertoire implies a large range of potential NK cell subpopulations (5). NK cells exhibit numerous functions other than cytotoxicity, including modulation of the behavior of other innate and adaptive immune cell populations, such as through cross-talk with dendritic cells (DCs) or cytokine production (6-10). In particular, NK cells strongly interact with DCs, resulting in the activation of both cell types $(11,12)$. Also, NK cells were shown to orientate the B cell response and the underlying affinity maturation via the restriction of follicular helper T cells, a feature that is key in the generation of broadly neutralizing antibodies (13). NK cell functions are influenced by vaccination and infection $(14,15)$. In addition, recent findings in mice, macaques, and humans indicate that NK cells show adaptive-like features (16-19). However, it is not yet fully clear how these findings can be exploited to improve the immunogenicity and protective efficacy of vaccines $(20,21)$. More studies on vaccine-induced NK cell immunity are thus admittedly required for improving vaccine design.

Modified vaccinia virus Ankara (MVA) is an attenuated vaccine derived from vaccinia virus, first developed as a vaccine against smallpox, which now serves as the vector for many recombinant vaccine candidates because of its capacity to induce strong and long-lasting 
immunity (22). MVA is known to activate NK cells in mouse bone marrow and spleen, where it induces NK cell proliferation (23), as well as in lymph nodes, where NK cells accumulate via CXCR3 signaling after being sensed by macrophages. Such recruitment is crucial for the induction of Th1 responses (24). Moreover, the vaccine we used (MVA HIV B) was reported to prime human NK cells via NK-DC crosstalk in vitro (25). Other studies in mice reported that NK cells are recruited to tissues in response to MVA-induced CCL2 (MCP-1) expression by macrophages (26). We previously reported a difference in the level of CCL2 in the blood of macaques after an MVA boost relative to prime (27). These and other studies show that MVA modulates NK cell activity and trafficking. They moreover suggest an important contribution of NK cells to MVA-induced immunity.

Nonhuman primates (NHP) are an important animal model in vaccinology, given their close immune proximity with humans, including innate immunity (28). Immune responses in macaques to human vaccine injection are highly predictive of vaccine immunogenicity in humans. This is particularly true for MVA (29). Human NK cells are usually subdivided based on CD56 and CD16 expression, whereas most NK cells from macaques are CD8 $\alpha^{+}$CD56 $^{-}(30)$. In addition, both human and macaque NK cells express NKp46, but in macaquesNKp46 may not be expressed by all NK cell subpopulations $(28,31)$. Nevertheless, close phenotypic analogies have been found between macaque and human NK cell subpopulations and functional studies have revealed similar behavior in both species (32-35).

We previously uncovered the phenotypic complexity and diversity of innate myeloid cells in the blood and the impact of vaccinations on the dynamics of their subset composition by mass cytometry (27) in cynomolgus macaques immunized with a recombinant MVA HIV-B. We used the very same animals and a similar analytical workflow, but a 31-marker mass cytometry 
96 antibody panel dedicated to the analysis of NK cells, to determine the phenotype of blood NK

97 cell subpopulations, as well as their diversity and evolution throughout the vaccination 98 process.

99 We demonstrated a high phenotypic diversity within the blood NK cell compartment in 100 macaques. Importantly, the study reveals the induction of changes within the NK cell 101 subphenotype composition by the prime, some of which were maintained over time. Hence, 102 the NK cells present at recall were different from those present at baseline. The key 103 phenotypic signature discriminating NK cells responding to boost from those responding to 104 prime was identified and included stronger expression of several cytotoxic, homing, and 105 adhesion molecules.

106 This study has important implications for understanding the role of NK cells in vaccine-induced 107 responses, as well as for the optimization of vaccine protocols. 


\section{Ethics statement}

110

111

112

The experimental protocols were approved by the ethics committee «Comité d'éthique en expérimentation animale $n^{\circ} 44 »$ under the reference 2015031314518254.02 (APAFIS\#319) for the longitudinal analysis of the MVA-induced response, and 2015062215324227v1 (APAFIS\#891) when comparing MVA and buffer injections, and the «Ministère de l'Education Nationale, de l'Enseignement Supérieur et de la Recherche» (France). Animals were handled by veterinary staff in accordance with national regulations (CEA Permit Number A 92-32-02) and the European Directive (2010/63, recommendation №9) and in compliance with the Standards for the Humane Care and Use of Laboratory Animals of the Office for Laboratory Animal Welfare (OLAW, USA) under OLAW Assurance number \#A5826-01.

\section{Experimental design}

Five male adult cynomolgus macaques, originating from Mauritius and identified as BB078, BB231, BC641, BD619, and BD620, were housed individually. Before inclusion in the study, they were tested to be negative for SIV, herpesvirus B, filovirus, STLV-1, SRV-1, SRV-2, measles virus, Hepatitis B antigen, and antibodies. Regarding CMV, BB078, BB231, and BC641 were seropositive, whereas BD620 was seronegative. Animals were captive-born (first generation, F1), 7-8 year old and weighed $8.2-10.7 \mathrm{~kg}$ at the beginning of the study.

Without prior selection, unbiased distribution of MHC haplotype was observed in our group of animals, with animals carrying one of the complete seven common haplotypes (with $\mathrm{H} 1, \mathrm{H} 2$, and $\mathrm{H} 3$ being the most common), or recombinants, and none of them being homozygous, or matching with one another for an entire MHC haplotype. 
Animals were inoculated subcutaneously with $4 \times 10^{8}$ PFU of the ANRS MVA HIV-B vaccine (MVATG17401; Transgene, Illkirch-Graffenstaden, France) (36), encoding HIV-Gag (aminoacids 1-519), Pol (amino-acids 172-219, 325-383 and 461-519), and Nef (amino-acids 66-147 and $182-206)$ proteins, as previously described $(27,37)$. Animals were immunized two months apart following a homologous prime-boost strategy.

For comparison of buffer and MVA injection, six macaques received a subcutaneous injection with a buffer containing $10 \mathrm{mM}$ Tris- $\mathrm{HCl}$, saccharose $5 \%(\mathrm{w} / \mathrm{v}), 10 \mathrm{mM} \mathrm{NaGlu}, 50 \mathrm{mM} \mathrm{NaCl}$, pH8.0. One month later they were inoculated with $4 \times 10^{8} \mathrm{PFU}$ of the ANRS MVA HIV-B vaccine. Animals were 2-5 year old and weighed 4.9 to $6.7 \mathrm{~kg}$. Without prior selection, unbiased distribution of MHC haplotype was observed in this control group of animals, with some animals carrying one of the complete seven common haplotypes or recombinants, and none of them being homozygous or matching with one another for an entire MHC haplotype.

Blood was collected in EDTA tubes for complete blood count and whole blood flow cytometry, lithium heparin tubes for whole blood mass cytometry analysis, and heparin cell preparation tube (CPT) (Becton Dickinson, Franklin Lakes, USA) for peripheral blood mononuclear cell (PBMC) isolation.

\section{Sample preparation}

Fixed leukocytes were prepared for mass cytometry using a previously described cell fixation protocol $(27,38,39)$, which allows the recovery of all leukocytes, including granulocytes, from lithium-heparin whole blood. Briefly, $1 \mathrm{~mL}$ of blood was incubated with a fixation buffer containing formaldehyde and glycerol for $10 \mathrm{~min}$ at $4^{\circ} \mathrm{C}$. After centrifugation, erythrocytes were lysed in $10 \mathrm{~mL}$ milli-Q water at room temperature for $20 \mathrm{~min}$. Cells were then washed in 
154

$1 X$ DPBS and stored at $-80^{\circ} \mathrm{C}$ at a final concentration of $15 \times 10^{6}$ cells $/ \mathrm{mL}$ in the fixation mixture. Note that cells were fixed extemporaneously without re-stimulation ex vivo.

PBMCs were prepared for transcriptome analysis. Blood was collected using CPT tubes. After centrifugation at $1,200 \times \mathrm{g}$ for $30 \mathrm{~min}$ at $\mathrm{RT}$, PBMCs were isolated, and remaining contaminating red blood cells, if any, were lysed with ACK lysis buffer (Thermo Fisher Scientific, Waltham, USA). PBMCs were then washed in complete culture medium composed of RPMI 1640 (Invitrogen, Carlsbad, USA) supplemented with 10\% heat-inactivated FCS (Eurobio, Courtaboeuf, France) and 1\% Penicillin-Streptomycin/Neomycin (Thermo Fisher Scientific, Waltham, USA).

\section{Cell staining and acquisition}

Fixed leukocyte staining and acquisition protocols were identical to those previously described (27). The 31-marker antibody panel used in the present study is described in Table 1. A number of classical NK cell receptors and other markers of interest $(6,40)$ could not be included at the time of the study due to lack of reactivity of the tested antibody clones with fixed macaque leukocytes (CD27: clones O323 and LG-7F9; NKp80: clones 4A4.D10 and 5D12; NKp30: clone AF29-4D12; CD117: clone 104D2; NKp46: clone BAB281; CX3CR1: clone 2A9-1; CXCR3: clone 1C6; CD122: clone Tu27; CD158a: clone HP-3E4; and CD161: clone DX12).

\section{Data processing and event selection}

FCS files were normalized with the MATLAB normalizer from Rachel Finck et al. (41). Replicates were concatenated using the tool from Cytobank (Mountain View, USA). Leukocytes were 
176 gated based on event length, iridium content, and exclusion of non-specifically stained

$177 \mathrm{CD}^{+} 6^{+} \mathrm{CD}^{+}$eosinophils $(27,39)$.

Complete blood count and cell population count

180

181

182

183

Complete blood counts (CBCs) were performed using blood collected in EDTA with the HmX instrument (Beckman Coulter). The absolute number of cells in each sample for a given cell population was computed as follows: $\mathrm{N}=$ the absolute number of leukocytes (expressed per $\mu \mathrm{L}$ of blood) $\mathrm{x}$ the number of cells in the population detected by CyTOF/total number of leukocytes (excluding $\mathrm{CD}^{+} \mathrm{CD}^{+} 6^{+}$cells) detected by CyTOF (given in Table 2).

\section{Identification of cell populations}

The Spanning-tree Progression Analyses of Density-normalized Events (SPADE) (42) algorithm was performed on the whole dataset of samples from macaques BB078, BB231, BC641 and BD620 to automatically identify cell populations displaying similar expression levels for the given markers used for clustering: CD66abce, HLA-DR, CD3, CD107a, CD8, CD45, granzyme B, CD56, CD62L, CD4, CD11a, CD2, CD7, NKG2D, CD11c, CD69, CD25, CD16, CCR5, CXCR4, CD14, perforin, NKG2A/C, CD20, and CCR7. Prior to clustering, we performed random predownsampling of 50,000 cells (corresponding to the highest number of cells contained in all samples) to avoid bias in the analysis towards samples with more cells than others (Table 2). The quality of the SPADE clustering, defined as a narrow and unimodal distribution for each marker in all cell clusters and NK cell clusters was assessed using the SPADEVizR R package we developed (43). 
Based on these quality control measurements, SPADE was parameterized to identify 900 clusters using a downsampling of $20 \%$, leading to $77.44 \%$ of all clusters with unimodal (Hartigan's dip test, $p$-value $\leq 0.05)$ and narrow distribution $(I Q R \leq 2)$ of all markers. Most of the non-unimodal distribution was attributable to perforin (154 clusters of 900 (17.11\%)).

Among NK cell clusters identified on the SPADE tree, based on CD3 and CD8 expression, $66.67 \%$ (22 of 33 ) had a unimodal and narrow distribution for all markers. Non-unimodal or wide distribution was not associated with a particular marker; at worst CD2 and CD16 expression were non-unimodal or wide for 4 of 33 clusters (12.12\%) (Table 3).

Two sets of baseline samples were available: 19 days before the prime (BPD19) and just before the prime, coded 0 hour post-prime (HOPP) in our nomenclature. Only BPD19 samples (available for the four macaques) were used for SPADE analysis to avoid biasing the SPADE analysis toward baseline samples, since NK cells were more numerous before than early after immunization. HOPP samples (only available for three of four animals) were upsampled into the SPADE analysis, using the closest neighborhood method. Briefly, cells from HOPP samples were assigned to the cluster of its closest cell-neighbor within the SPADE analysis. The neighborhood definition was based on the SPADE clustering markers. The closest neighbors were found using the FNN R package (available at https://CRAN.R-project.org/package=FNN) and the kd-tree approach.

Samples from macaque BD619 were not included in this SPADE analysis since only H3PP, H6PP, D1PP, and D1PB samples were available. Indeed, samples from this animal would have been underrepresented among the samples from the other animals, and they could have biased the clustering analysis towards early modification of the NK subphenotype composition. Nevertheless, BD619 samples were mapped afterwards onto the SPADE tree for phenotypic 
characterization based on the same closest neighbor approach used to map HOPP baseline samples.

We directly identified blood NK cells on the SPADE tree based on CD3 and CD8 expression, rather than by manual gating followed by SPADE analysis of the NK cells to avoid a bias in the manual gating of $\mathrm{CD}^{-} \mathrm{CD} 8^{+}$events and contamination with $\mathrm{CD}^{\text {low }}$ neutrophils, which displayed a low background signal in all channels including CD8.

\section{Categorical heatmap representation of NK cell clusters phenotypes}

The median expression among all samples was used to generate the categorical heatmap using SPADEVizR (43). The range of marker expression was divided into five categories between the $5^{\text {th }}$ and the $95^{\text {th }}$ percentiles to define the cell cluster phenotype. Samples containing less than 10 cells were removed from the median computation. Hierarchical clustering, represented by the cluster and marker dendrograms in the heatmap, was performed using the Euclidean metric and the ward.D linkage method. The cluster dendrogram was used to define phenotypic families.

\section{LASSO-LDA model to classify post-prime and post-boost NK cell immune profiles}

The classification of post-prime and post-boost NK cell immune profiles was performed using a combination of the Least Absolute Shrinkage and Selection Operator (LASSO) and Linear Discriminant Analysis (LDA) methods. The LASSO method was based on the lars R package (available at https://CRAN.R-project.org/package=lars). Abundance profiles of phenotypic families were centered and reduced. Model validity was assessed through the leave-one-out 
cross-validation method. The best configuration was chosen using the elbow criterion. Essentially, the minimum number of phenotypic families was chosen such that adding more phenotypic families did not improve the model. Graphically, this corresponds to the number of phenotypic families for which a break in slope (an "elbow") is observed when plotting the mean square error of the model as a function of the number of phenotypic families used (Figure S5B). The LDA method was based on the MASS R package (available at https://CRAN.Rproject.org/package=MASS). Marker expression density distributions were compared using the CytoCompare R package (44) based on the Kolmogorov-Smirnov distance.

\section{Validation of the LASSO-LDA model}

The LASSO-LDA classifier generated using BB078, BB231, BC641, and BD620 samples was used to classify BD619 samples and validate the model. Cell cluster abundances from BD619 samples were centered and reduced with the abundance of the four other animals.

\section{RNA extraction and gene expression profiling}

PBMCs were cultured overnight at $2.5 \times 10^{6} \mathrm{PBMCs} /$ well in U-bottom 96-well plates (BD Falcon) in duplicate. PBMCs were recovered and lysed in $350 \mu \mathrm{L}$ of RLT Plus buffer (Qiagen, Hilden Germany) with $1 \%$ of mercaptoethanol. RNA was then purified using the RNeasy Plus Micro Kit (Qiagen, Hilden Germany). Purified RNA was quantified using an ND-8000 spectrophotometer (NanoDrop Technologies, Fisher Scientific, Illkirch, France) and the integrity verified on a 2100 BioAnalyzer (Agilent Technologies, Massy, France). cDNA was synthesized and biotin-labeled using Ambion Illumina TotalPrep RNA Amplification kits 
265 (Applied Biosystem/Ambion, Saint-Aubin, France). Labeled cRNA was hybridized to Illumina

266 Human HT-12V4 BeadChips, previously successfully used to analyze cynomolgus macaque 267 whole genome $(45,46)$. All steps were performed following the manufacturers' protocols.

\section{Transcriptomic analysis}

Transcriptomic signals were background corrected and quantile-normalized using the limma R package (available at https://bioconductor.org/packages/release/bioc/html/limma.html).

We identified genes associated with NK cell abundance (which is relatively low among PBMCS) by performing a two-step analysis approach. First, genes for which the expression correlated with total NK cell abundance (Pearson correlation, $|R| \geq 0.65$ and $p \leq 0.05$ ) were analyzed using the STRING database (47) to define interaction networks.

Transcriptomic data were expressed as fluorescence intensity (resulting from DNA probe hybridization) per $2.5 \times 10^{6}$ PBMCs. The percentage of NK cells among PBMCs was used for the correlation between transcript expressions and NK cell abundances. The number of PBMC in each leukocyte sample was estimated in our mass cytometry analysis by excluding $\mathrm{CD}^{+} 6^{+}$cells. Thus, the percentage of NK cells among PBMCs was defined as the number of $\mathrm{CD}^{-}{ }^{-} \mathrm{CD} 8^{+} \mathrm{NK}$ cells detected in the CyTOF analysis divided by the number of $\mathrm{CD}^{-} 6^{-}$leukocytes identified in the CyTOF analysis multiplied by 100 . The Pearson coefficient of correlation was used to quantify the association based on log-transformed data. The transcriptomic timepoint D57PP was associated with the mass cytometry timepoint HOPB (corresponding to D58PP). In addition, early transcriptomic timepoints $(H 3, H 6, D 1)$ were missing for both immunizations. The transcriptomic timepoints used were: D-19PP, D3PP, D57PP, and D3PB. 
Second, genes having interactions with at least one other gene were selected, and a functional enrichment analysis was performed using Ingenuity Pathways Analysis software (Ingenuity Systems, Inc, IPA, Redwood City, USA) to further decipher the gene signature. IPA maps each gene identifier to its corresponding molecule in the Ingenuity Pathways Knowledge Base (IPKB). For all analyses, $\mathrm{p}$-values generated by Fisher's exact test were adjusted by BenjaminiHochberg Multiple Testing.

\section{Correlation between NK cell and innate myeloid cell dynamics}

The Spearman correlation coefficient between the abundance (number of cells per $\mathrm{mL}$ ) of blood NK cell phenotypic families and the abundance of blood innate myeloid cell kinetic families (groups of phenotypic families sharing similar dynamics as previously defined (27)) was computed. The correlation was considered significant when $|R| \geq 0.6$ and $p \leq 0.05$.

\section{Area under the curve}

Areas under the curve (AUC) were calculated as the cumulative sum of concentrations of the population between $\mathrm{H} 0$ and D3 (either after prime or after boost). Post-prime and post-boost AUC were compared using the permutation test from the exactRankTests R package (available at https://cran.r-project.org/web/packages/exactRankTests/index.html).

\section{Inter-individual variability}


The inter-individual variability in term of phenotypic composition was quantified as the percentage of NK cells that are not classified in the same phenotypic families between two animals.

\section{Flow cytometry}

Flow cytometry staining was used to identify NK cells in a control group of 6 macaques used to assess whether the effect of immunizations on NK cell number was specific of MVA subcutaneous injection or could be induced by the sole buffer subcutaneous injection or no injection (only anesthetic). For each sample, $100 \mu \mathrm{L}$ of blood were stained during 30 minutes with $90 \mu \mathrm{L}$ of mix of antibodies diluted in BD Horizon ${ }^{\circledR}$ stained buffer (BD Biosciences, Franklin Lakes, USA) containing CD123 (BD Biosciences, Franklin Lakes, USA, Clone 7G3), HLA-DR (BD Biosciences, Franklin Lakes, USA, clone G46-6), CD163 (BD Biosciences, Franklin Lakes, USA, clone GHI/61), CD11c (Biolegend, San Diego, CA, USA clone 3.9), CD45 (BD Biosciences, Franklin Lakes, USA, clone D058-1283), CD66 (Miltenyi Biotec, Bergisch Gladbach, Germany, clone TET2), CD3 (BD Biosciences, Franklin Lakes, USA, clone SP34-2), CD20 (BD Biosciences, Franklin Lakes, USA, clone 2H7), CD8 (BD Biosciences, Franklin Lakes, USA, clone RPA-T8), CD11b (Beckman Coulter, Brea, USA, clone Bear 1), CD14 (BD Biosciences, Franklin Lakes, USA, Clone M5E2), CD33 (Miltenyi Biotec, Bergisch Gladbach, Germany, Clone AC104.3E3), CD16 (Beckman Coulter, Brea, USA, 3G8) and NKG2A (Beckman Coulter, Brea, USA, clone Z199), and then cells were fixed and red blood cells were removed with $1 \mathrm{~mL}$ of BD FACs Lysing ${ }^{\circledast}$ (BD Biosciences, Franklin Lakes, USA) during 10 minutes at room temperature and washed twice using PBS. Samples were acquired with a BD LSR Fortessa (BD Biosciences, Franklin Lakes, USA). 
NK cells were gated as CD66- $\mathrm{CD}^{-} \mathrm{CD}^{-} \mathrm{CD}^{-} \mathrm{C}^{-} \mathrm{CD}^{+}$cells using FlowJo 9 software (FlowJo, Ashland, USA). The absolute count numbers were calculated as the percentage of NK cells

332 among all cells $X$ leukocyte count (CBC).

\section{Data availability}

Gated cytometry profiles are available on the FlowRepository database (48) under accession number FR-FCM-ZYPY. Raw transcriptomic profiles are available on the EBI-ArrayExpress database (49) under accession number E-MTAB-7697. Main graphical representations and statistical results are available in an interactive format on the IDMIT data dissemination platform accessible at http://data.idmitcenter.fr/MVA-innate-NK/. 


\section{Results}

342

\section{Total NK cell kinetics do not differ between prime and boost}

We vaccinated four adult male cynomolgus macaques with a recombinant MVA-based vaccine following the homologous prime-boost strategy described in Figure1A $(27,37)$. Blood samples were taken before and at various timepoints during the vaccination time course and fixed extemporaneously without ex vivo re-stimulation with the vaccine. All samples were stained with the antibody panel targeting markers of NK cell activation (e.g., CD25 and CD69), function (e.g., IFNץ, perforin, granzyme $\mathrm{B}, \mathrm{CD} 107 \mathrm{a}$, and $\mathrm{CD} 11 \mathrm{a}$ ), and maturation (e.g., CD2, CD7, and CXCR4), described in Figure 1B and detailed in Table 2. We then followed the analysis pipeline described in Figure 1C. Preliminary analyses showed high inter-individual variability in terms of NKG2A/C expression among NK cells, not associated with CMV serology (Figure S1).

First, we performed a SPADE analysis to identify cell populations based on the expression of the following markers: CD66abce, HLA-DR, CD3, CD107a, CD8, CD45, granzyme B, CD56, CD62L, CD4, CD11a, CD2, CD7, NKG2D, CD11c, CD69, CD25, CD16, CCR5, CXCR4, CD14, perforin, NKG2A/C, CD20, and CCR7. This strategy allowed the segregation of NK cells, defined classically for macaques as $\mathrm{CD}^{-} \mathrm{CD}^{+}$cells, from other leukocytes and into 33 cell clusters on a separate branch of the SPADE tree (Figure 2A). Note that we notably excluded CD66 ${ }^{+}$ neutrophils, $\mathrm{HLA}_{-\mathrm{DR}}{ }^{+} \mathrm{CD} 14^{+}$monocytes, $\mathrm{HLA}^{-\mathrm{DR}}{ }^{+} \mathrm{CD} 11 \mathrm{c}^{+} \mathrm{CDCs}, \mathrm{CD}^{+} \mathrm{T}$ cells, and HLA-DR ${ }^{+}$ $\mathrm{CD} 20^{+} \mathrm{B}$ cells (Figure S2).

We analyzed the kinetics of all NK cell clusters in the blood throughout vaccination (Figure 2B). As expected, the number of total NK cells in the blood was relatively low $<<$ $0.5 \times 10^{6}$ cells $/ \mathrm{mL}$ for all timepoints). NK cell numbers were homogeneously affected by immunization in all animals. Indeed, NK cell numbers tended to decrease between $3 \mathrm{~h}(\mathrm{H} 3)$ 
and $6 \mathrm{~h} / 1$ day (H6-D1) post-immunization, both post-prime (PP) and post-boost (PB). These changes in NK cell counts were likely to be MVA injection-specific, as shown by six additional control animals (Figure S3). A high variability in term of NK cell count was observed between baselines (untreated animals, before buffer injection, and before MVA injection) across the control animals (Figure S3A). Buffer injection did not induce a significant early decrease in total NK cell count $(p=0.9839)$ (Figure S3B), whereas MVA injection did $(p=0.03697)$ (Figure S3C).

This MVA-induced decrease in NK cell count likely corresponds to NK cell recruitment to either inflamed tissues or lymphoid organs, consistent with a previous study in mice describing the recruitment of NK cells to the draining lymph node within the first day after MVA injection (24). There were no significant differences in the NK cell dynamics between the two immunizations based on the comparison of post-prime and post-boost areas under the curves.

To further complete the picture of the NK cell response at the whole compartment level, we analyzed the gene signature associated with NK cell number using microarrays on isolated PBMCs (Figure 3A). There were numerous genes for which the expression correlated with NK cell abundance with a remarkably high number of gene interactions, among them, a cluster of NK cell-associated genes, such as activation/cytotoxic associated molecules (CD226, CD69, KLRK1 (NKG2D), granzyme B, and granzyme H) (50-53), as well as genes encoding proteins involved in these signaling pathways (notably ITGAL/CD11a and VAV3) (54) and the chemoattractant cytokine CCL27 (55).

Functional enrichment of this interacting network of genes confirmed a strong association with NK cells, which indicated that we were able to find an NK cell-associated signature within the PBMCs. More specifically, this gene signature was associated with NK cell/DC crosstalk, 
NK cell cytotoxic activity, and FcR-mediated phagocytosis. To a lesser extent, this signature was associated with granulocyte diapedesis (a surprising signature, as only PBMC RNA was used) and fatty acid oxidation (Figure 3B). Among regulators, the most statistically significant was the IL-12 complex (Figure 3C), which is a well-known key cytokine in NK cell biology (56). ESR1, LCK, CD46, and ITGAL/CD11a were also found to be engaged. ESR1, LCK, and ITGAL/CD11a are associated with NK cell cytotoxic activity $(54,57-59)$, whereas CD46 is associated with complement activity (60).

We previously showed, with the very same animals that IL-12 production was upregulated in blood neutrophils responding to the second MVA injection, as compared to those responding to the first MVA inoculation. IL-12 concentration in plasma did not differ between prime and boost though. (27). Thus, since no significant difference was found at the level of total NK cell number by contrast to IL-12 level in neutrophils, and since IL-12 signaling appeared correlated with NK cell number in blood, we further investigated whether differences could exist at a deeper phenotypic resolution of the NK cell compartment.

\section{The NK cell compartment displays numerous subphenotypes}

We used high-dimensional analysis based on marker expression intensity to investigate potential changes in NK cell phenotype after immunization. The double clustering of markers and NK cell clusters resulted in a categorical heatmap with a marker and cell cluster dendrogram. It facilitated the visualization of the phenotypes of all NK cell clusters at a glance

(Figure 4). On this heatmap, marker expression was divided into five bins between the $5^{\text {th }}$ and $95^{\text {th }}$ percentile of the distribution across the whole dataset. This allowed us to qualify the 
expression of each marker for each cluster as very low, low, mid, high or very high, according to the bin in which the cluster fell for the indicated marker.

The hierarchical clustering of markers represented in the marker dendrogram revealed two large groups of co-expressed markers, further subdivided into four subgroups (Figure 4). One large group of co-expressed markers contained cytotoxic and maturation markers and comprised highly co-expressed markers among most NK cells (granzyme B, CD107a, NKG2A/C, $\mathrm{CD} 8, \mathrm{CD} 7, \mathrm{CD} 45$, and CD11a) in a first subgroup and highly/moderately co-expressed markers (perforin, NKG2D, CD16, CD2, CCR5, CD56, and CXCR4) in a second. The second large group contained, notably, several cytokine and chemokine receptors and consisted of moderately/weakly co-expressed markers (IL-10, Ki-67, MIP-1 $\beta$, IL-4, TNF $\alpha$, CD20, CD69, and CD11c) in a first subgroup and weakly/unexpressed markers (CD14, CD4, HLA-DR, CD25, CD62L, IFNY, CCR7, CD66, and CD3) in a second.

The hierarchical clustering of NK cell clusters, represented in the cluster dendrogram, revealed 10 phenotypic NK cell families, arbitrarily numbered from 1 to 10, distributed within three superfamilies, named A to C (Figure 4). Each phenotypic family contained NK cell clusters with similar phenotypes, and each superfamily was composed of proximal phenotypic families. Phenotypic families are likely to better correspond to biologically meaningful cell populations than cell clusters. Indeed, the number of leukocyte clusters chosen as an entry parameter in our SPADE analysis (900 cell clusters) was optimally defined to achieve a uniform and narrow expression of all clustering markers in a maximum number of leukocyte clusters. Admittedly, it could have resulted in a potentially artificially high number of NK cell clusters ( 33 NK cell clusters). The risk of over-clustering was overcome by merging phenotypically similar NK cell clusters into phenotypic families. 
Superfamily A (phenotypic families $5,3,8$, and 1 ) consisted of NKG2 ${ }^{\text {high }}$ CD16 $6^{\text {high }}$ CD107a ${ }^{\text {high }}$

434 CCR5 $^{\text {high }}$ NK cells. Superfamily B (phenotypic families 4,2 , and 6) consisted of NKG2D ${ }^{\text {mid }}$ $435 \mathrm{CD}_{16} 6^{\text {mid }} \mathrm{CD} 107 \mathrm{a}^{\text {high }}$ CCR5 ${ }^{\text {mid }}$ NK cells. Finally, superfamily $\mathrm{C}$ (phenotypic families 10,7 , and 9) 436 contained NKG2D ${ }^{\text {low }}$ CD16 ${ }^{\text {low }}$ CD107a ${ }^{\text {mid }}$ CCR5 $5^{\text {low }}$ NK cells.

Beyond such wide phenotypic NK cell diversity, which underlined varying degrees of expression of cytotoxicity markers and likely past, ongoing or future cytotoxicity, two phenotypic families (phenotypic families 1 and 4 ) in superfamilies A and B were CD2 $2^{\text {high }}$, 440 suggesting higher activation ability towards antibody coated pathogen/antigen binding. CD2 is a well-known NK cell activator (61), which was shown to potentiate the CD16 signaling cascade in vivo in humans (62). This action was later shown to be associated with CD58 engagement on infected cells by $\mathrm{CD} 2^{+} \mathrm{NK}$ cells in vitro (63). Also, phenotypic family 9 , within superfamily $C$, was the only one to be $C D 7^{\text {mid }}$ (all other NK clusters were $C D 7^{\text {high }}$ ), likely related 445 to a lower maturity. Indeed CD7 has been shown to be expressed on highly differentiated cytotoxic and cytokine-producing NK cells ex vivo in humans (64). Moreover, two phenotypic

447 families containing one single cluster displayed very peculiar phenotypes (Figure S4). Phenotypic family 8, was CD66 ${ }^{\text {high }}$, whereas other NK cell clusters were CD66 low/-, as expected. It may consist of activated NK cells that can be inhibited through CD66, as reported after homotypic CD66a interactions between melanoma and NK cells (65). Phenotypic family 10 was HLA-DR ${ }^{\text {high }}$ granzyme $B^{\text {low }}$ CD107alow and may correspond to "NK DCs" observed in mouse 452 tissues (66) and ex vivo in humans (67). 
455 We then studied the dynamics of all identified NK cell phenotypic families (Figure 5A), which, 456 for some, contrasted with those of total NK cells (Figure 2B). We identified distinct and 457 complex patterns.

458 The phenotypic family 7 was more highly affected by the prime than the boost (AUC 459 comparison, $\mathrm{p}=0.0286)$.

By contrast, two phenotypic families ( 3 and 8 ) were more highly affected by the boost than the prime (AUC comparison, $p=0.057$ and 0.0286 , respectively). In particular, family 3 showed a strong increase at H3PB compared to H3PP $(p=0.0286)$.

The remaining seven phenotypic families $(1,2,4,5,6,9$ and 10) displayed strong interindividual variability in their dynamics and various patterns. Phenotypic family 10 notably only showed a very low peak at D14PP. Still note that for phenotypic family 5, 3 animals out of 4 showed a stronger increase in number post-prime than post-boost.

Although the number of total NK cells was low throughout vaccination and essentially transiently decreased (Figure 2B), many NK cell subphenotypes (e.g., phenotypic families 7, 3, 5,1 , and 9) conversely showed an increase in absolute number for some timepoints (Figure 5A).

We then determined the relative abundance of the phenotypic families within each animal for 472 each timepoint (Figure 5B). There was high inter-individual diversity of the NK cell compartment relative to that of the innate myeloid cell compartment (27). In addition, NK cell number and composition strongly differed between both baseline samples at D-19PP and HOPP, in the absence of any other experimental perturbation of the immune system (Figure S5). At baseline, prior to any immunization, there was high intra-individual variability in terms

477 of cell number, with a difference of up to $0.8 \times 10^{6} \mathrm{NK}$ cells $/ \mathrm{mL}$ of blood between D19PP and 
HOPP(Figure S5). This is fully consistent with the results obtained on the six additional control animals (Figure S3A). To note, the phenotypic composition was remarkably stable within each animal between the two baselines (Figure S5).

Note that prior to any immunization a high inter-individual variability was observed in term of the phenotypic composition of the NK cell compartment. Actually, on average $50 \pm 6 \%$ of the NK cell compartment differ phenotypically between two animals (i.e., $50 \pm 6 \%$ of NK cells were associated with distinct phenotypic families between two animals). Strikingly, the phenotypic composition of the NK cell compartment after immunization was far more similar between the different animals ( $32 \pm 6 \%$ of difference between individuals at every other timepoint), indicating that immunization homogenizes the NK cell compartment composition.

The distribution of NK cell subphenotypes changed markedly throughout the prime, as early as H3PP, and dramatically between H6PP and D1PP. The major shift in the composition of the NK cell compartment remained relatively stable up to D14 (Figure 5B). Further changes of the subphenotype composition occurred later, between D14PP and the boost (at D58PP = H0PB). This was not observed for innate myeloid cells, for which the shift occurred essentially between D14PP and the boost (27). One explanation is that these subphenotypes correspond to newly generated immature NK cells arising from the bone marrow, whereas all NK cells expressing homing markers, such as CCR5, CCR7, CD62L, and CXCR4 (which is indeed the case for families 3, 5, and 8, which decreased in frequencies at these timepoints) were previously recruited to tissues. Consistent with this hypothesis, the major phenotypic families at these timepoints belonged to superfamily C of poorly-cytotoxic NK cells (Figure 4). In addition, this switch may also reflect the persistence of some poorly cytotoxic NK cells in the blood after vaccination, rather than a true increase in number or redistribution. For example, family 7 
501

502

503

504

505

506

507

508

509

510

511

512

513

514

515

516

517

518

519

520

521

522

remained constant in number at D1PP but still became proportionally one the most abundant families at this timepoint, because of the decrease in the numbers of the other NK cell populations (Figure 5A and 5B).

Overall, this analysis demonstrated that the NK cell compartment was modified by the priming immunization, and the NK cell subphenotypes composition was not similar at HOPB relative to that at baseline. Strikingly, NK cells were mainly phenotypically highly cytotoxic at HOPB (phenotypic families 3 and 8 ), compared to HOPP samples where most NK cells were poorly/moderately cytotoxic (Figure 4 and Figure 5B). Note that this phenotype modification occurred before the boosting immunization and is thus independent of the boost.

We finally analyzed the diversity and dynamics of the NK cell compartment using the Simpson index as a readout (Figure 5C). The wide diversity of the NK cell receptor repertoire, for which each combination of NK cell receptors can virtually give rise to a new subset of NK cells, was recently uncovered (5). However, the meaning of such NK cell diversity for vaccines is not yet understood. Admittedly, the lack of a larger set of inhibitory and activating NK receptors (which are difficult to analyze in NHP) in our antibody panel prevented us from directly addressing the issue of the NK cell repertoire, for which the diversity was previously shown to reflect immune experience (68). Nonetheless, we were able to observe two distinct and complex kinetic patterns among our four animals (BB078 and BB231 vs. BC641 and BD620), but by no means did we detect a progressive increase in NK cell subphenotype diversity over time and after immunization.

Altogether, the mass cytometry analysis revealed that the prime induced the modification of the NK cell subphenotype composition in two main steps, at D1PP and between D14PP and 
the boost (D58PP). As a result of these phenotypic differences pre-existing prior to the boost, the NK cell response differed between prime and boost.

\section{Key phenotypic signatures between the NK cell response to prime and boost}

We then aimed to define the NK cell phenotypic families that discriminate the primary and secondary NK cell responses using an approach that combined LASSO and LDA methods.

We first used the LASSO method to determine the optimal number of phenotypic families that could account for post-prime and post-boost differences (Figure S6). Based on this analysis, we chose phenotypic families $3,8,7,5$, and 6 that were necessary and sufficiently informative to distinguish prime and boost samples through leave-one-out cross-validation (Figure S6). These five phenotypic families were then used to build the LDA classification (Figure 6A and 6B). The classification of post-prime and post-boost samples was correct for 31 of 33 samples (94\%) and showed that phenotypic families 5 and 7 were involved with the post-prime response, whereas phenotypic families 3,6 , and 8 were involved with the post-boost response.

We further determined the phenotypic differences that distinguished NK cells that responded to the prime from those responding to the boost. We examined the mean signal intensity (MSI) of prime-responding vs. boost-responding NK cells and identified eight markers that differed in expression intensity between the two signatures (Figure 6C and 6D): granzyme B, CD107a, perforin, CD69, CD66abce, CCR5, CD11c, and CD16. All were more highly expressed after the boost than the prime. To a lesser extent, CD11a was also more highly expressed after the boost than the prime (Figure 6D). This suggests that NK cells involved with the post-boost immune response showed a more cytotoxic phenotype (including the ability for antibody- 
dependent cell cytotoxicity-ADCC- based on CD16 expression), associated with an increased ability to traffic to lymph nodes and inflamed tissues. Phenotypic family 5 , which was involved in the post-prime response, also belonged to superfamily A of highly cytotoxic NK cells, together with phenotypic families 3 and 8 (Figure 4). However, it displayed a higher CCR5 expression and lower levels of $\mathrm{CD} 2, \mathrm{CD} 7, \mathrm{CD} 16$, and CD11a than the post-boost highly cytotoxic NK cells from phenotypic families 3 and 8, suggesting a stronger ability to traffic to inflamed tissues, while simultaneously showing a less mature/activated phenotype. This observation is consistent with the fact that NK cells responding to the boost showed a more cytotoxic (and potentially more mature) phenotype in the blood than those responding to the prime.

\section{Validation of the phenotypic signature distinguishing NK responses to prime and boost}

To validate the results and model obtained on samples from four animals, we used four samples from a fifth animal from the same cohort, macaque BD619, which was not included in the previous steps of the analysis. After associating each cell to the SPADE cluster it was the closest to, we were able to define the phenotypic composition of these samples with respect to our SPADE analysis (Figure 7A). BD619 showed a phenotypic signature fairly consistent with the four animals used to build the model, with a high abundance of phenotypic family 7 and 9 at H3PP, H6PP and D1PP, a high abundance of phenotypic families 2 and 4 at D1PP and D1PB, and high abundance of phenotypic family 3 at D1PB. We then applied the LDA classifier detailed in Figure 6 on these new samples (Figure 7B). Three samples (H3PP, D1PP, and D1PB) out of four were correctly classified. Interestingly, the sole error made was on H6PP (which obtained a low post-boost score), the timepoint for which the model already misclassified the 
BC641 sample. This misclassification is due to the relatively high abundance of family 8 at H6PP for these two animals, while family 8 is overall more enriched after the boost and was used as such in the LDA classifier.

In conclusion, applying our SPADE analysis and resulting LDA generated from "only" four animals (but 39 samples) on those previously unseen samples gave consistent results and strengthened the definition of our NK cell signature to prime and boost.

\section{The NK cell response correlates with the innate myeloid response}

We further investigated how the NK cell response integrated with the innate myeloid response to MVA. We previously reported, in the same animals, that neutrophils, monocytes, and cDCs responded differently to the priming and boosting immunization. Some subphenotypes were enriched only after one of the two immunizations, with cells responding to the boost expressing higher levels of markers involved in phagocytosis, antigen presentation, costimulation, chemotaxis, and inflammation (27). Here, we assessed the correlation between the dynamics of NK cell subphenotypes and those of these innate myeloid cell subphenotypes, based on cell abundance (Figure 8).

The abundance of NK cells responding to the prime inversely correlated $(R<-0.6)$ with that of the innate myeloid cells responding to the boost, but did not correlate $(|R|<0.6)$ with that of innate myeloid cells responding to the prime. In contrast, the abundance of NK cells responding to the boost positively correlated $(R>0.6)$ with that of innate myeloid cells responding to the boost, but there was no association $(|R|<0.6)$ with that of innate myeloid cells responding to the prime. One explanation may be that the kinetics of NK cells and innate myeloid cells are not synchronous. Indeed, NK cell expansion mainly occurred at $\mathrm{H} 3-\mathrm{H} 6$ for the 
592 post-prime expanded subphenotypes, whereas innate myeloid cells that expanded post-prime 593 were still numerous at D1. Conversely, innate myeloid cells that expanded post-boost mainly 594 expanded around $\mathrm{H6}$, simultaneously with NK cells. This also indicates that innate myeloid and 595 NK cell responses are more synchronous during the response to the boost. 


\section{Discussion}

We previously reported that vaccination elicits a distinct innate myeloid immunity between prime and after boost (27). Using the very same animals, we show here that, NK cell immune responses also differ between each immunization. In contrast to the myeloid response, blood NK cell dynamics were mainly driven by decreases in cell number and there was wider interindividual variability. We have previously documented a transient decrease of NK cell numbers in the blood after intradermal MVA injection in macaques (46). In contrast to total NK cells, some NK cell subphenotypes increased in number after immunization, with some showing a differential enrichment after the prime and boost. More strikingly, our study revealed that some modifications of NK cell subphenotype composition towards a more mature and cytotoxic phenotype were induced by the prime. These changes occurred in two steps : a first early and quite long-lasting shift in phenotype (from D1PP and maintained up to D14PP), followed by a later one (between D14PP and D58PP). As a core result of these phenotypic changes, the NK cell composition before the prime and prior to the boost differed, and the NK cells responding to the boost were phenotypically more mature/cytotoxic.

There are numerous terminologies used in literature to described distinct NK cells subpopulations displaying memory-like features including but not limited to antigen-specific NK cells $(17-19,69)$, cytokine-induced NK cells $(18,19)$, cytokines activated NK cells $(69)$, liverrestricted NK cells (19), memory-like NK cells (18) or adaptive NK cells (18). Many of these data were obtained in patients or animals infected by the cytomegalovirus. To our knowledge there is not yet a clear consensus on the phenotype of these different subpopulations of NK cells, although some markers seem to be important, such as Ly49H and KLRG1 in mice or NKG2C and CD57 in humans $(19,69)$. Due to a lack of reactivity with fixed macaque cells of antibodies 
targeting many of NK receptors (such as NKp80, NKp46, NKp30, and CD158a), as well as CD57, those markers could not be included in our analyses, and available antibodies could not distinguish between NKG2A and NKG2C in macaques. In the present study, the main argument for induction of memory-like NK cells is the emergence or preponderance of some particular NK subphenotypes and overall their persistence long after MVA prime (2 months). Whether these MVA prime induced NK cells correspond to memory-like NK cells, and which one (cytokine-induced or antigen-specific), remain to be fully tested with functional assays and transcriptional profiling approaches.

In addition, these missing markers may impair the capture of the whole NK cell diversity and explain why our LDA classifier was less efficient when dealing with NK cell subpopulation to distinguish post-prime and post-boost samples than the LDA generated on innate myeloid cells subpopulations (27), despite strong correlations between NK cell and innate myeloid cell response at the boost.

Besides, we noticed a high inter-individual variability in NK cell counts and phenotype at steady state, prior to any immunization, while this inter-individual variability was low after immunization, with all four animals behaving similarly. This indicated that changes induced by vaccination went beyond the sole inter-individual variability. In other words at baseline, without stimulation, the NK cells compartment activity is highly variable, whereas, upon stimulation (e.g., immunization, very likely infection), this variability decreases. The number of animals $(n=4)$ was not sufficient to address in details the variation in the phenotypic composition of the NK cell compartment prior to immunization. This would be a valuable problematic to tackle with, since this may explain some of the inter-individual differences in 
641

642 cancer).

643

644

645 related genes in PBMCs, in particular with the involvement of IL-12. However, the lack of

646 available transcriptomic data at very early timepoints (such as H3, H6, and D1 post-

647 immunization) prevented us from further assessing the kinetics of these transcripts during this

648 period in which many dramatic changes in the abundance of NK cell phenotypic families

649

650

651

652

653

654

655

656

657

658

659

660

661

662

663

Interestingly, the total NK cell dynamics were associated with the modulation of NK cell-

occurred. In addition, transcript levels were assessed at the PBMC level, with NK cells potentially overwhelmed by B cells, T cells, and monocytes. Overall this transcriptomic dataset was rather a complementary piece of evidence to show that immunizations impacted NK cells as a cellular compartment. Future studies addressing vaccine-induced NK cell response should include early timepoints and use purified NK cells or even single cells, given the wide diversity of the NK cell repertoire. Still, interestingly, IL-12 has been described to be required in the efficient differentiation of both antigen-specific memory NK cells and cytokines activated NK cells in mice model after MCMV infection (69). Since we previously showed that IL-12 was upregulated in neutrophils in our vaccine schedule, we may hypothesize that IL-12 could play a similar role in the development of the phenotypically modified NK cells we observed here.

We used mass cytometry to identify key markers that clearly distinguish the NK cell immune response to the prime from that to the boost. Granzyme B, CD107a, perforin, CD69, CD66, CCR5, CD11C, CD16, and, to a lesser extent, CD11a were upregulated by NK cells responding to the boost. Several hypotheses can explain these prime-boost differences among NK cells, notably the presence of primary circulating antibodies that could activate NK cells via FcRs 
664 (such as CD16), as well as the local immune reaction involving primary memory $\mathrm{T}$ cells and/or 665 imprinting resident macrophages. But actually, these differences were induced by the prime 666 and pre-existed to the boost.

667 CD107a, CD11a, CD11c, CD69, CD16, granzyme B, and perforin are associated with NK cell 668 maturation and cytotoxic activity $(53,54,70-72)$, suggesting that NK cells would be more 669 cytotoxic after the boost than after the prime. The increase in CCR5 expression suggests that 670 NK cells are more prone to recruitment to inflamed tissues, including the injection site or 671 draining lymphoid organs $(73,74)$. CD66 inhibits NKG2D signaling and subsequent cytotoxicity 672 and may thus play a role in the downregulation of inflammation $(65,75)$, consistent with the 673 overall milder inflammation observed in the macaques at the boost relative to the prime (27). 674 This suggests that prime-induced modifications of the NK cell compartment likely make it 675 more effective in responding to subsequent infection/immunization, given its high activation 676 potential. Whether direct ligand/receptor interactions between MVA and NK cells or cytokines 677 play a role remains to be determined. Also, functional analyses are required to define the 678 exact enhanced functions of the phenotypically distinct NK cells responding to the boost. Deep 679 phenotyping analyses were performed on these animals on different cell compartments, not 680 only in this paper but also elsewhere $(27,37)$. As a consequence, the number of blood samples 681 left available was too limited to assess NK cell functions at relevant timepoints.

682 Our results are in contrast to those obtained in mice, showing that MVA immunization, as 683 opposed to vaccinia virus immunization, failed to induced memory-like NK cells after a single intraperitoneal injection (76). This strongly suggests that live, replication-competent micro685 organisms are likely to be more efficient at priming innate immune memory. Several 686 hypotheses could explain the discrepancies between these results in mice and ours, apart 
687

688

689

690

691

692

693

694

695

696

697

698

699

700

701

702

703

704

705

706

707

708

709

from the simple difference between animal models. Indeed, different routes of injection may differentially influence systemic immunity. For example, previous studies on trained immunity showed that intravenous, but not subcutaneous, injection of BCG induced stem cells gave rise to trained myeloid progeny (77). The impact that the route of injection could have on NK cell responses is still largely unaddressed (21). Another explanation is that the authors focused on the NK cell compartment six months after priming, without analyzing intermediate timepoints. It is possible that the primed NK cells we observed in our setting may be only short-lived and would vanish in the long-term in the absence of boosting.

Another question is whether those primed NK cells were antigen-specific (and in this case MVA or HIV) or not. Indeed, one may wonder in which extent these phenotypically modified NK cells provided cross-protection to a wide range of pathogens, as for trained innate myeloid cells (78) or whether they would be restricted to some specific antigens (18). Indeed, should they be antigen-specific, they would respond differently to the boost only if the correct antigen is present in the boosting immunization. Should they be non-antigen-specific (cytokine-induced memory NK cells), they would likely respond differently irrespectively of the boost. Further functional studies will be required to firmly conclude on antigen specificity.

Strikingly, CD16, CCR5, and in a lesser extent CD11a, were more highly upregulated on NK cells after the boost than the prime, similarly to monocytes, DCs, and neutrophils (27). This indicates that (i) these features are shared by both lymphoid and myeloid innate cells and (ii) one consequence of a boost is more consistent CCR5 upregulation, which is likely linked to tissue recruitment. However, the innate myeloid and NK cell responses were clearly distinct, with innate myeloid cells being rapidly enriched after immunization, whereas NK cell numbers decreased. In addition, the kinetics of subphenotype composition modifications induced by 
the prime differed between NK and innate myeloid cells. This suggests that the mechanisms behind the training of innate lymphoid and myeloid immune cells differ.

We found strong correlations between NK cell and innate myeloid cell responses. Responses to the boost clearly correlated between the two compartments. Similarly, the innate myeloid response to the boost negatively correlated with that of the NK cells to the prime. In contrast, the innate myeloid response to the prime did not correlate with that of the NK cells to the prime, neither did it negatively correlate with the NK cell response to the boost. Overall, this suggests that the innate response to the boost is more coordinated between NK cells and innate myeloid cells than the response to the prime. Whether this is the result of a more efficient crosstalk between NK cells and myeloid cells after the boost than after the prime is yet to be addressed.

Finally, addressing whether some features of the NK cell response correlate with the adaptive immune response and how this could be used to better predict the establishment of immune memory is still a challenge. Also, further investigating NK cell responses in tissues other than blood is another challenge that will need to be met to obtain a fully comprehensive picture of the vaccine-induced NK cell response.

To our knowledge, this is the first study using CyTOF technology for the longitudinal analysis of NK cells after vaccination. It revealed key features of NK cell phenotype after immunization and without any ex vivo re-stimulation with the vaccine in contrast to other studies analyzing the 'recall' NK cell response $(8,79,80)$. This work aims to pave the way for future studies aiming to exploit this knowledge to optimize future vaccine. 


\section{Authorship}

Conceptualization: RLG and ASB; immunization and blood sampling: NDB; cytometry: JLP, NT, $\mathrm{NH}, \mathrm{JEY}, \mathrm{AC}, \mathrm{MMT}$, and ASB; flow cytometry for MVA-buffer comparison: PR, FM, JLP, NT, and ASB; transcriptomics: JLP, NT, CL, HH, and ASB; writing of the original draft: JLP, NT, ASB; review and editing: $\mathrm{NH}, \mathrm{JEY}, \mathrm{CL}, \mathrm{PR}, \mathrm{NDB}, \mathrm{FM}, \mathrm{HH}, \mathrm{AC}, \mathrm{MMT}, \mathrm{YL}$, and RLG; acquisition of funding: YL, RLG, and ASB; supervision: RLG and ASB.

\section{Acknowledgments}

This work was supported by the "Investissements d'Avenir" programs managed by the ANR under reference ANR-10-LABX-77-01, funding the Vaccine Research Institute (VRI), Créteil (ImMemory research program), ANR-11-INBS-0008, funding the Infectious Disease Models and Innovative Therapies (IDMIT, Fontenay-aux-Roses, France) infrastructure, and ANR-10EQPX-02-01, funding the FlowCyTech facility (IDMIT, Fontenay-aux-Roses, France), and by the European Union EVHA H2020 project (Grant N681032). We would like to thank all members of the ASW and L2I groups from IDMIT as well as Romain Marlin. Nicolas Tchitchek held fellowships from the ANRS (France Recherche Nord\&Sud Sida-HIV Hépatites).

\section{Conflict of interest disclosure}

The authors declare no conflict of interest. 
1. Iwasaki A, Medzhitov R. Regulation of adaptive immunity by the innate immune system. Science (2010) 327:291-295. doi:10.1126/science.1183021

2. Artis D, Spits H. The biology of innate lymphoid cells. Nature (2015) 517:293-301. doi:10.1038/nature14189

3. Eberl G, Di Santo JP, Vivier E. The brave new world of innate lymphoid cells. Nat Immunol (2015) 16:1-5. doi:10.1038/ni.3059

4. Boudreau JE, Hsu KC. Natural Killer Cell Education and the Response to Infection and Cancer Therapy: Stay Tuned. Trends Immunol (2018) 39:222-239. doi:10.1016/j.it.2017.12.001

5. Wilk AJ, Blish CA. Diversification of human NK cells: Lessons from deep profiling. J Leukoc Biol (2018) 103:629-641. doi:10.1002/JLB.6RI0917-390R

6. Vivier E, Tomasello E, Baratin M, Walzer T, Ugolini S. Functions of natural killer cells. Nat Immunol (2008) 9:503-510. doi:10.1038/ni1582

7. Pampena MB, Levy EM. Natural killer cells as helper cells in dendritic cell cancer vaccines. Front Immunol (2015) 6:13. doi:10.3389/fimmu.2015.00013

8. Horowitz A, Behrens RH, Okell L, Fooks AR, Riley EM. NK cells as effectors of acquired immune responses: effector $\mathrm{CD} 4+\mathrm{T}$ cell-dependent activation of NK cells following vaccination. J Immunol (2010) 185:2808-2818. doi:10.4049/jimmunol.1000844

9. Gao N, Jennings $P$, Yuan D. Requirements for the natural killer cell-mediated induction of IgG1 and IgG2a expression in B lymphocytes. Int Immunol (2008) 20:645-657. doi:10.1093/intimm/dxn021

10. Crouse J, Xu HC, Lang PA, Oxenius A. NK cells regulating $T$ cell responses: mechanisms and outcome. Trends Immunol (2015) 36:49-58. doi:10.1016/j.it.2014.11.001

11. Cooper MA, Fehniger TA, Fuchs A, Colonna M, Caligiuri MA. NK cell and DC interactions. Trends Immunol (2004) 25:47-52.

12. Van Elssen CHMJ, Oth T, Germeraad WTV, Bos GMJ, Vanderlocht J. Natural killer cells: the secret weapon in dendritic cell vaccination strategies. Clin Cancer Res (2014) 20:1095-1103. doi:10.1158/1078-0432.CCR-13-2302

13. Rydyznski CE, Cranert SA, Zhou JQ, Xu H, Kleinstein SH, Singh H, Waggoner SN. Affinity Maturation Is Impaired by Natural Killer Cell Suppression of Germinal Centers. Cell Rep (2018) 24:33673373.e4. doi:10.1016/j.celrep.2018.08.075

14. Vargas-Inchaustegui DA, Ying O, Demberg T, Robert-Guroff M. Evaluation of Functional NK Cell Responses in Vaccinated and SIV-Infected Rhesus Macaques. Front Immunol (2016) 7:340. doi:10.3389/fimmu.2016.00340

15. Costanzo MC, Kim D, Creegan M, Lal KG, Ake JA, Currier JR, Streeck H, Robb ML, Michael NL, Bolton DL, et al. Transcriptomic signatures of NK cells suggest impaired responsiveness in HIV-1 
infection and increased activity post-vaccination. Nat Commun (2018) 9:1212. doi:10.1038/s41467-018-03618-w

16. Sun JC, Ugolini S, Vivier E. Immunological memory within the innate immune system. EMBO J (2014) 33:1295-1303. doi:10.1002/embj.201387651

17. Reeves RK, Li H, Jost S, Blass E, Li H, Schafer JL, Varner V, Manickam C, Eslamizar L, Altfeld M, et al. Antigen-specific NK cell memory in rhesus macaques. Nat Immunol (2015) 16:927-932. doi:10.1038/ni.3227

18. Paust S, Blish CA, Reeves RK. Redefining Memory: Building the Case for Adaptive NK Cells. J Virol (2017) 91: doi:10.1128/JVI.00169-17

19. Min-Oo G, Kamimura Y, Hendricks DW, Nabekura T, Lanier LL. Natural killer cells: walking three paths down memory lane. Trends Immunol (2013) 34:251-258. doi:10.1016/j.it.2013.02.005

20. Jost S, Altfeld M. Control of human viral infections by natural killer cells. Annu Rev Immunol (2013) 31:163-194. doi:10.1146/annurev-immunol-032712-100001

21. Rydyznski CE, Waggoner SN. Boosting vaccine efficacy the natural (killer) way. Trends Immunol (2015) 36:536-546. doi:10.1016/j.it.2015.07.004

22. Volz A, Sutter G. Modified Vaccinia Virus Ankara: History, Value in Basic Research, and Current Perspectives for Vaccine Development. Adv Virus Res (2017) 97:187-243. doi:10.1016/bs.aivir.2016.07.001

23. Milo I, Blecher-Gonen R, Barnett-Itzhaki Z, Bar-Ziv R, Tal O, Gurevich I, Feferman T, Drexler I, Amit I, Bousso P, et al. The bone marrow is patrolled by NK cells that are primed and expand in response to systemic viral activation. Eur J Immunol (2018) doi:10.1002/eji.201747378

24. Garcia Z, Lemaître F, van Rooijen N, Albert ML, Levy Y, Schwartz O, Bousso P. Subcapsular sinus macrophages promote NK cell accumulation and activation in response to lymph-borne viral particles. Blood (2012) 120:4744-4750. doi:10.1182/blood-2012-02-408179

25. Moreno-Nieves UY, Didier C, Lévy Y, Barré-Sinoussi F, Scott-Algara D, ANRS HIV Vaccine Network (AHVN). NK cells are primed by ANRS MVA(HIV)-infected DCs, via a mechanism involving NKG2D and membrane-bound IL-15, to control HIV-1 infection in CD4+ T cells. Eur J Immunol (2014) 44:2370-2379. doi:10.1002/eji.201344149

26. Lehmann MH, Torres-Domínguez LE, Price PJR, Brandmüller C, Kirschning CJ, Sutter G. CCL2 expression is mediated by type I IFN receptor and recruits NK and T cells to the lung during MVA infection. J Leukoc Biol (2016) 99:1057-1064. doi:10.1189/jlb.4MA0815-376RR

27. Palgen J-L, Tchitchek N, Elhmouzi-Younes J, Delandre S, Namet I, Rosenbaum P, DereuddreBosquet N, Martinon F, Cosma A, Lévy Y, et al. Prime and Boost Vaccination Elicit a Distinct Innate Myeloid Cell Immune Response. Sci Rep (2018) 8:3087. doi:10.1038/s41598-018-21222-2

28. Hong HS, Rajakumar PA, Billingsley JM, Reeves RK, Johnson RP. No monkey business: why studying NK cells in non-human primates pays off. Front Immunol (2013) 4:32. doi:10.3389/fimmu.2013.00032

29. Davies DH, Wyatt LS, Newman FK, Earl PL, Chun S, Hernandez JE, Molina DM, Hirst S, Moss B, Frey $\mathrm{SE}$, et al. Antibody profiling by proteome microarray reveals the immunogenicity of the 
attenuated smallpox vaccine modified vaccinia virus ankara is comparable to that of Dryvax. $J$ Virol (2008) 82:652-663. doi:10.1128/JVI.01706-07

30. Carter DL, Shieh TM, Blosser RL, Chadwick KR, Margolick JB, Hildreth JE, Clements JE, Zink MC. CD56 identifies monocytes and not natural killer cells in rhesus macaques. Cytometry (1999) 37:41-50.

31. Sivori S, Vitale M, Morelli L, Sanseverino L, Augugliaro R, Bottino C, Moretta L, Moretta A. p46, a novel natural killer cell-specific surface molecule that mediates cell activation. J Exp Med (1997) 186:1129-1136.

32. Huot N, Jacquelin B, Garcia-Tellez T, Rascle P, Ploquin MJ, Madec Y, Reeves RK, DerreudreBosquet N, Müller-Trutwin M. Natural killer cells migrate into and control simian immunodeficiency virus replication in lymph node follicles in African green monkeys. Nat Med (2017) 23:1277-1286. doi:10.1038/nm.4421

33. Vargas-Inchaustegui DA, Helmold Hait S, Chung HK, Narola J, Hoang T, Robert-Guroff M. Phenotypic and Functional Characterization of Circulatory, Splenic, and Hepatic NK Cells in Simian Immunodeficiency Virus-Controlling Macaques. J Immunol (2017) 199:3202-3211. doi:10.4049/jimmunol.1700586

34. Webster RL, Johnson RP. Delineation of multiple subpopulations of natural killer cells in rhesus macaques. Immunology (2005) 115:206-214. doi:10.1111/j.1365-2567.2005.02147.x

35. Reeves RK, Evans TI, Gillis J, Johnson RP. Simian immunodeficiency virus infection induces expansion of alpha4beta7+ and cytotoxic CD56+ NK cells. J Virol (2010) 84:8959-8963. doi:10.1128/JVI.01126-10

36. Brandler S, Lepelley A, Desdouits M, Guivel-Benhassine F, Ceccaldi P-E, Lévy Y, Schwartz O, Moris A. Preclinical studies of a modified vaccinia virus Ankara-based HIV candidate vaccine: antigen presentation and antiviral effect. J Virol (2010) 84:5314-5328. doi:10.1128/JVI.02329-09

37. Pejoski D, Tchitchek N, Rodriguez Pozo A, Elhmouzi-Younes J, Yousfi-Bogniaho R, Rogez-Kreuz C, Clayette P, Dereuddre-Bosquet N, Lévy Y, Cosma A, et al. Identification of Vaccine-Altered Circulating B Cell Phenotypes Using Mass Cytometry and a Two-Step Clustering Analysis. J Immunol (2016) 196:4814-4831. doi:10.4049/jimmunol.1502005

38. Egger G, Burda A, Glasner A. A simple method for measuring the F-actin content of human polymorphonuclear leukocytes in whole blood. Virchows Arch (2001) 438:394-397.

39. Elhmouzi-Younes J, Palgen J-L, Tchitchek N, Delandre S, Namet I, Bodinham CL, Pizzoferro K, Lewis DJM, Le Grand R, Cosma A, et al. In depth comparative phenotyping of blood innate myeloid leukocytes from healthy humans and macaques using mass cytometry. Cytometry $A$ (2017) doi:10.1002/cyto.a.23107

40. Montaldo E, Del Zotto G, Della Chiesa M, Mingari MC, Moretta A, De Maria A, Moretta L. Human NK cell receptors/markers: a tool to analyze NK cell development, subsets and function. Cytometry A (2013) 83:702-713. doi:10.1002/cyto.a.22302

41. Finck R, Simonds EF, Jager A, Krishnaswamy S, Sachs K, Fantl W, Pe'er D, Nolan GP, Bendall SC. Normalization of mass cytometry data with bead standards. Cytometry A (2013) 83:483-494. doi:10.1002/cyto.a.22271 
42. Qiu P, Simonds EF, Bendall SC, Gibbs KD, Bruggner RV, Linderman MD, Sachs K, Nolan GP, Plevritis SK. Extracting a cellular hierarchy from high-dimensional cytometry data with SPADE. Nat Biotechnol (2011) 29:886-891. doi:10.1038/nbt.1991

43. Gautreau G, Pejoski D, Le Grand R, Cosma A, Beignon A-S, Tchitchek N. SPADEVizR: an R package for Visualization, Analysis and Integration of SPADE results. Bioinformatics (2016) doi:10.1093/bioinformatics/btw708

44. Platon L, Pejoski D, Gautreau G, Targat B, Le Grand R, Beignon A-S, Tchitchek N. A computational approach for phenotypic comparisons of cell populations in high-dimensional cytometry data. Methods (2017) doi:10.1016/j.ymeth.2017.09.005

45. Marlin R, Nugeyre M-T, Tchitchek N, Parenti M, Hocini H, Benjelloun F, Cannou C, DereuddreBosquet N, Levy Y, Barré-Sinoussi F, et al. Modified Vaccinia Virus Ankara Vector Induces Specific Cellular and Humoral Responses in the Female Reproductive Tract, the Main HIV Portal of Entry. $\mathrm{J}$ Immunol (2017) 199:1923-1932. doi:10.4049/jimmunol.1700320

46. Rosenbaum P, Tchitchek N, Joly C, Stimmer L, Hocini H, Dereuddre-Bosquet N, Beignon A-S, Chapon C, Levy Y, Le Grand R, et al. Molecular and Cellular Dynamics in the Skin, the Lymph Nodes, and the Blood of the Immune Response to Intradermal Injection of Modified Vaccinia Ankara Vaccine. Front Immunol (2018) 9: doi:10.3389/fimmu.2018.00870

47. Szklarczyk D, Morris JH, Cook H, Kuhn M, Wyder S, Simonovic M, Santos A, Doncheva NT, Roth A, Bork $P$, et al. The STRING database in 2017: quality-controlled protein-protein association networks, made broadly accessible. Nucleic Acids Res (2017) 45:D362-D368. doi:10.1093/nar/gkw937

48. Spidlen J, Breuer K, Rosenberg C, Kotecha N, Brinkman RR. FlowRepository: a resource of annotated flow cytometry datasets associated with peer-reviewed publications. Cytometry $A$ (2012) 81:727-731. doi:10.1002/cyto.a.22106

49. Kolesnikov N, Hastings E, Keays M, Melnichuk O, Tang YA, Williams E, Dylag M, Kurbatova N, Brandizi M, Burdett T, et al. ArrayExpress update--simplifying data submissions. Nucleic Acids Res (2015) 43:D1113-1116. doi:10.1093/nar/gku1057

50. Martinet L, Ferrari De Andrade L, Guillerey C, Lee JS, Liu J, Souza-Fonseca-Guimaraes F, Hutchinson DS, Kolesnik TB, Nicholson SE, Huntington ND, et al. DNAM-1 expression marks an alternative program of NK cell maturation. Cell Rep (2015) 11:85-97. doi:10.1016/j.celrep.2015.03.006

51. Biassoni R, Fogli M, Cantoni C, Costa P, Conte R, Koopman G, Cafaro A, Ensoli B, Moretta A, Moretta L, et al. Molecular and functional characterization of NKG2D, NKp80, and NKG2C triggering NK cell receptors in rhesus and cynomolgus macaques: monitoring of NK cell function during simian HIV infection. J Immunol (2005) 174:5695-5705.

52. Borrego F, Robertson MJ, Ritz J, Peña J, Solana R. CD69 is a stimulatory receptor for natural killer cell and its cytotoxic effect is blocked by CD94 inhibitory receptor. Immunology (1999) 97:159165.

53. Trapani JA, Smyth MJ. Functional significance of the perforin/granzyme cell death pathway. Nat Rev Immunol (2002) 2:735-747. doi:10.1038/nri911 
54. Urlaub D, Höfer K, Müller M-L, Watzl C. LFA-1 Activation in NK Cells and Their Subsets: Influence of Receptors, Maturation, and Cytokine Stimulation. J Immunol (2017) 198:1944-1951. doi:10.4049/jimmunol.1601004

55. Gao J-Q, Tsuda Y, Han M, Xu D-H, Kanagawa N, Hatanaka Y, Tani Y, Mizuguchi H, Tsutsumi Y, Mayumi T, et al. NK cells are migrated and indispensable in the anti-tumor activity induced by CCL27 gene therapy. Cancer Immunol Immunother (2009) 58:291-299. doi:10.1007/s00262-0080554-x

56. Zwirner NW, Ziblat A. Regulation of NK Cell Activation and Effector Functions by the IL-12 Family of Cytokines: The Case of IL-27. Front Immunol (2017) 8: doi:10.3389/fimmu.2017.00025

57. Barber DF, Faure M, Long EO. LFA-1 contributes an early signal for NK cell cytotoxicity. J Immunol (2004) 173:3653-3659.

58. Kovats S. Estrogen receptors regulate innate immune cells and signaling pathways. Cell Immunol (2015) 294:63-69. doi:10.1016/j.cellimm.2015.01.018

59. Brumbaugh KM, Binstadt BA, Billadeau DD, Schoon RA, Dick CJ, Ten RM, Leibson PJ. Functional Role for Syk Tyrosine Kinase in Natural Killer Cell-mediated Natural Cytotoxicity. J Exp Med (1997) 186:1965-1974.

60. Cardone J, Le Friec G, Kemper C. CD46 in innate and adaptive immunity: an update. Clin Exp Immunol (2011) 164:301-311. doi:10.1111/j.1365-2249.2011.04400.x

61. McNerney ME, Kumar V. The CD2 family of natural killer cell receptors. Curr Top Microbiol Immunol (2006) 298:91-120.

62. Liu LL, Landskron J, Ask EH, Enqvist M, Sohlberg E, Traherne JA, Hammer Q, Goodridge JP, Larsson $\mathrm{S}$, Jayaraman J, et al. Critical Role of CD2 Co-stimulation in Adaptive Natural Killer Cell Responses Revealed in NKG2C-Deficient Humans. Cell Rep (2016) 15:1088-1099. doi:10.1016/j.celrep.2016.04.005

63. Rölle A, Halenius A, Ewen E-M, Cerwenka A, Hengel H, Momburg F. CD2-CD58 interactions are pivotal for the activation and function of adaptive natural killer cells in human cytomegalovirus infection. Eur J Immunol (2016) 46:2420-2425. doi:10.1002/eji.201646492

64. Milush JM, Long BR, Snyder-Cappione JE, Cappione AJ, York VA, Ndhlovu LC, Lanier LL, Michaëlsson J, Nixon DF. Functionally distinct subsets of human NK cells and monocyte/DC-like cells identified by coexpression of CD56, CD7, and CD4. Blood (2009) 114:4823-4831. doi:10.1182/blood-2009-04-216374

65. Markel G, Lieberman N, Katz G, Arnon TI, Lotem M, Drize O, Blumberg RS, Bar-Haim E, Mader R, Eisenbach L, et al. CD66a interactions between human melanoma and NK cells: a novel class I MHC-independent inhibitory mechanism of cytotoxicity. J Immunol (2002) 168:2803-2810.

66. Pillarisetty VG, Katz SC, Bleier JI, Shah AB, Dematteo RP. Natural killer dendritic cells have both antigen presenting and lytic function and in response to $\mathrm{CpG}$ produce IFN-gamma via autocrine IL-12. J Immunol (2005) 174:2612-2618.

67. Zingoni A, Sornasse T, Cocks BG, Tanaka Y, Santoni A, Lanier LL. Cross-talk between activated human NK cells and CD4+ T cells via OX40-OX40 ligand interactions. J Immunol (2004) 173:37163724. 
68. Strauss-Albee DM, Fukuyama J, Liang EC, Yao Y, Jarrell JA, Drake AL, Kinuthia J, Montgomery RR, John-Stewart G, Holmes $S$, et al. Human NK cell repertoire diversity reflects immune experience and correlates with viral susceptibility. Sci Transl Med (2015) 7:297ra115. doi:10.1126/scitranslmed.aac5722

69. Nabekura T, Lanier LL. Tracking the fate of antigen-specific versus cytokine-activated natural killer cells after cytomegalovirus infection. J Exp Med (2016) 213:2745-2758. doi:10.1084/jem.20160726

70. Bruhns P, Jönsson F. Mouse and human FcR effector functions. Immunol Rev (2015) 268:25-51. doi:10.1111/imr.12350

71. Burt BM, Plitas G, Stableford JA, Nguyen HM, Bamboat ZM, Pillarisetty VG, DeMatteo RP. CD11C identifies a subset of murine liver natural killer cells that responds to adenoviral hepatitis. $J$ Leukoc Biol (2008) 84:1039-1046. doi:10.1189/jlb.0408256

72. Aranami T, Miyake S, Yamamura T. Differential expression of CD11c by peripheral blood NK cells reflects temporal activity of multiple sclerosis. J Immunol (2006) 177:5659-5667.

73. Khan IA, Thomas SY, Moretto MM, Lee FS, Islam SA, Combe C, Schwartzman JD, Luster AD. CCR5 is essential for NK cell trafficking and host survival following Toxoplasma gondii infection. PLoS Pathog (2006) 2:e49. doi:10.1371/journal.ppat.0020049

74. Weiss ID, Shoham H, Wald O, Wald H, Beider K, Abraham M, Barashi N, Galun E, Nagler A, Peled A. Ccr5 deficiency regulates the proliferation and trafficking of natural killer cells under physiological conditions. Cytokine (2011) 54:249-257. doi:10.1016/j.cyto.2011.01.011

75. Mizrahi S 'ar, Yefenof E, Gross M, Attal P, Ben Yaakov A, Goldman-Wohl D, Maly B, Stern N, Katz G, Gazit R, et al. A phenotypic and functional characterization of NK cells in adenoids. J Leukoc Biol (2007) 82:1095-1105. doi:10.1189/jlb.0407205

76. Gillard GO, Bivas-Benita M, Hovav A-H, Grandpre LE, Panas MW, Seaman MS, Haynes BF, Letvin NL. Thy1+ NK [corrected] cells from vaccinia virus-primed mice confer protection against vaccinia virus challenge in the absence of adaptive lymphocytes. PLoS Pathog (2011) 7:e1002141. doi:10.1371/journal.ppat.1002141

77. Kaufmann E, Sanz J, Dunn JL, Khan N, Mendonça LE, Pacis A, Tzelepis F, Pernet E, Dumaine A, Grenier J-C, et al. BCG Educates Hematopoietic Stem Cells to Generate Protective Innate Immunity against Tuberculosis. Cell (2018) 172:176-190.e19. doi:10.1016/j.cell.2017.12.031

78. Netea MG, van der Meer JWM. Trained Immunity: An Ancient Way of Remembering. Cell Host Microbe (2017) 21:297-300. doi:10.1016/j.chom.2017.02.003

79. Long BR, Michaelsson J, Loo CP, Ballan WM, Vu B-AN, Hecht FM, Lanier LL, Chapman JM, Nixon DF. Elevated frequency of gamma interferon-producing NK cells in healthy adults vaccinated against influenza virus. Clin Vaccine Immunol (2008) 15:120-130. doi:10.1128/CVI.00357-07

80. Darboe A, Danso E, Clarke E, Umesi A, Touray E, Wegmuller R, Moore SE, Riley EM, Goodier MR. Enhancement of cytokine-driven NK cell IFN- $\gamma$ production after vaccination of HCMV infected Africans. Eur J Immunol (2017) 47:1040-1050. doi:10.1002/eji.201746974 
987

988

989

990

991

992

993

994

995

996

997

998

999

1000

1001

1002

1003

1004

1005

1006

1007

1008

1009

\section{Figure legends}

Figure 1. Experimental design and analysis strategy. The experimental approach, including (A) the vaccine schedule, (B) the 31-marker antibody panel, and (C) the analysis pipeline, is shown. Five cynomolgus macaques were subcutaneously immunized with MVA-HIV B vaccine (4.10 8 PFU) twice, two months apart. Blood samples were collected at the indicated timepoints, hours $(H)$ or days $(D)$, post-prime (PP), and post-boost (PB), for mass cytometry (blue dots) or transcriptomic experiments (green dots). FCS files retrieved from mass cytometry were entered into the SPADE algorithm after exclusion of background events, dead cells, and non-specifically stained putative eosinophils, to cluster leukocytes based on 25 clustering markers and identify NK cell clusters. NK cell clusters were further clustered into phenotypic families whose kinetics were analyzed. Prime vs. boost phenotypic signatures were eventually determined using the LASSO-LDA approach.

Figure 2. Identification of blood NK cells. (A) The generated SPADE tree is shown. This analysis was built using all samples from the dataset, except HOPP samples, which were later mapped onto the analysis. NK cell clusters $\left(\mathrm{CD}^{-} \mathrm{CD} 8^{+}\right)$are indicated in red. (B) The absolute number of total NK cells per individual animal at each timepoint is shown. Red arrows indicate MVA immunization. The mean AUC +/- SD is indicated, as well as the $p$-value, after comparison by the permutation test and considered statistically significant when $p \leq 0.05$.

Figure 3. NK cell-associated transcriptomic signature. (A) Interactions among transcripts for which the expression correlated with NK cell abundance identified using the STRING database (47) $(|R| \geq 0.65$, p-value $\leq 0.05)$. (B) Top five canonical pathways correlating with NK cell 
1010

1011

1012

1013

1014

1015

1016

1017

1018

1019

1020

1021

1022

1023

1024

1025

1026

1027

1028

1029

1030

1031

abundance identified using IPA (Ingenuity Systems, Inc). (C) Top five upstream regulators correlating with NK cell abundance identified using IPA (Ingenuity Systems, Inc).

Figure 4. Phenotypic heatmap of NK cells. Each line of the heatmap corresponds to one cell cluster and each column to one marker. Marker expression is displayed according to phenotypical categorical bins, corresponding to the subdivision of marker range of expression in five categories between the $5^{\text {th }}$ and $95^{\text {th }}$ percentile of expression (the color code is indicated). The marker and cluster dendrograms are shown on the top and left, respectively.

The cluster dendrogram defined phenotypic families and superfamilies. Phenotypic families were randomly numbered and colored with different shades of the same color for each superfamily. Markers used as SPADE clustering makers are shown in bold.

Figure 5. Different enrichment of NK cells after each immunization. (A) The individual abundance in the number of cells/ $\mu \mathrm{L}$ of blood of each phenotypic family is shown over time. The mean AUC \pm standard deviation and p-value (permutation test) are shown and the phenotypic families are grouped based on their profiles. (B) The composition of the phenotypic families is indicated over time for each timepoint and each animal. The size of the pie is proportional to the absolute count of total NK cells in the blood, as indicated. Pie slices correspond to phenotypic families and are color-coded as in Fig. 4. na stands for not available. (C) The inverse Simpson index, as a readout for diversity, is displayed for each animal over time. Each color represents a distinct animal. 
1032

1033

1034

1035

1036

1037

1038

1039

1040

1041

1042

1043

1044

1045

1046

1047

1048

1049

1050

1051

1052

1053

1054

Figure 6. Signature distinguishing the post-prime and post-boost NK cell response. LDA was performed after LASSO regression to select the combination of phenotypic families that best discriminate between post-prime (blue, phenotypic families 5 and 7) and post-boost (red, phenotypic families 6,3 , and 8) samples. Note that the baseline samples (HOPP and HOPB) were not used for this analysis. (A) LDA coefficients and (B) LDA scores for each sample are shown. (C) MSI histograms for the eight markers with the highest Kolmogorov Smirnov distance between phenotypic families that best discriminate between the post-prime (blue) and the post-boost (red) responses as defined in (Fig. 6A) are displayed based on the merge of all samples and all timepoints. (D) The Kolmogorov Smirnov distance of all markers, including the top eight markers with the highest distance (bright gray vs. dark gray) between phenotypic families that best discriminate between the post-prime and the post-boost responses are displayed.

Figure 7. Validation of the NK cell signature after immunization(s). Samples from macaque BD619 were mapped into the existing SPADE tree to $(A)$ define the phenotypic composition of the NK cells compartment of these samples, and (B) assess the quality of the LDA model using new samples in the model generation.

Figure 8. Intercorrelation between NK cell and innate myeloid cell immune responses. Correlations between the abundance of NK and innate myeloid cell subphenotypes (27) were computed using the Spearman method. Correlations with $|R| \geq 0.6$ and $p \leq 0.05$ are represented by green $(R \geq 0.6)$ and purple $(R \leq 0.6)$ lines joining the indicated subphenotypes. LASSO/LDA was used to discriminate post-prime (blue) and post-boost (red) NK cell (Fig. 6A) 
1055 and innate myeloid cell subphenotypes (27). Subphenotypes not necessary for the 1056 classification are shown in black.

1057 


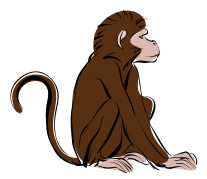

Cynomolgus macaque

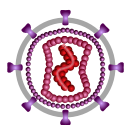

MVA HIV B

\section{NK cells phenotype}

Poorly cytotoxic Moderately cytotoxic Highly cytotoxic
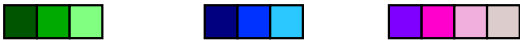

Abundant NK cells Heterogeneously cytotoxic

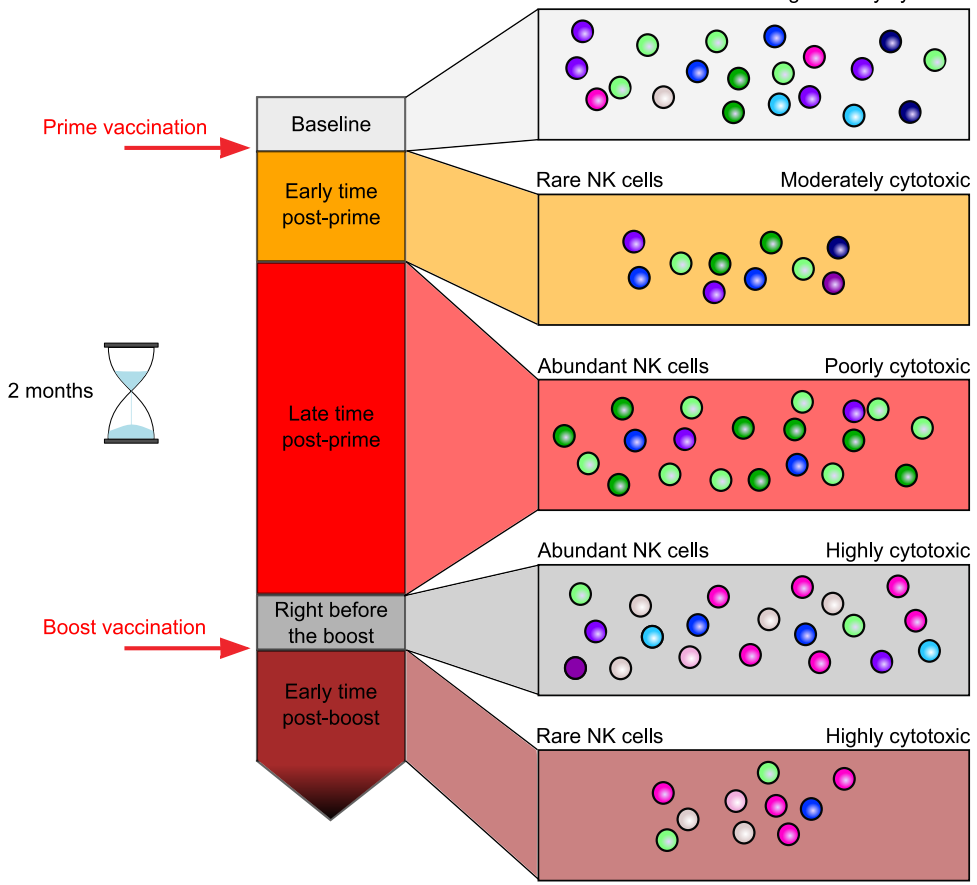


A

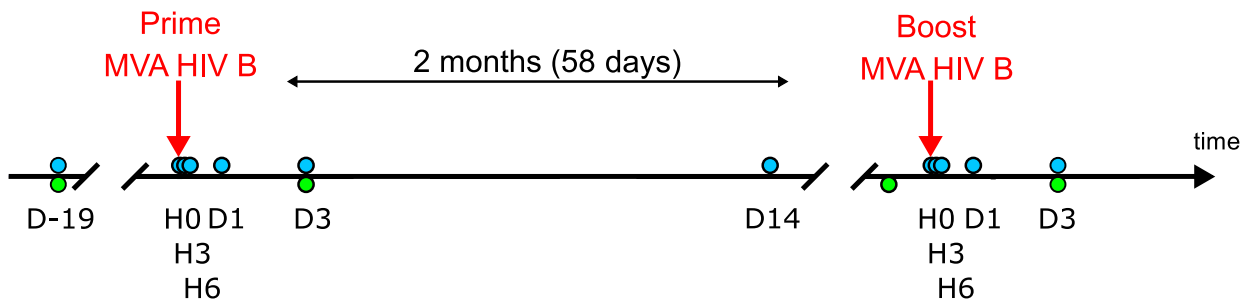

B

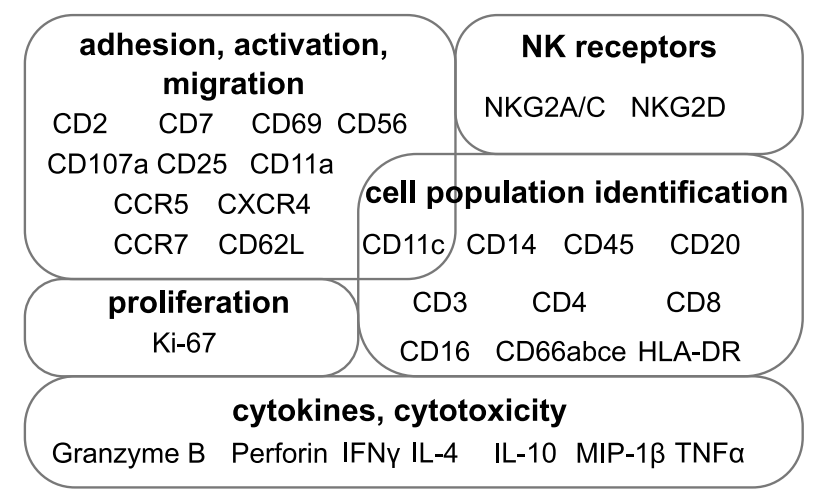

C

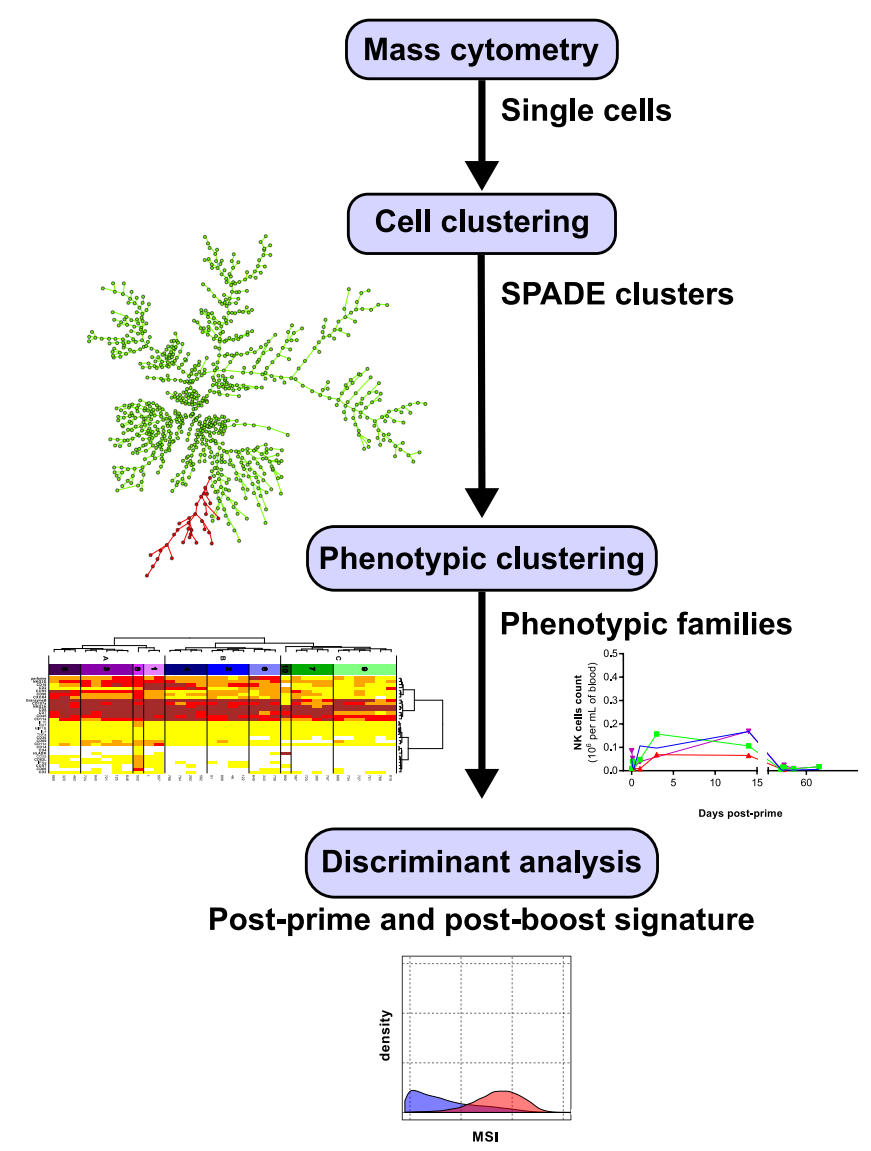


A

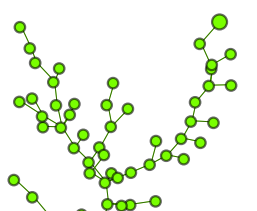

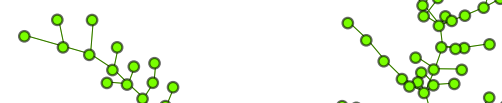

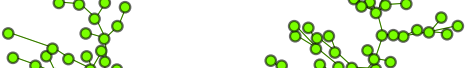

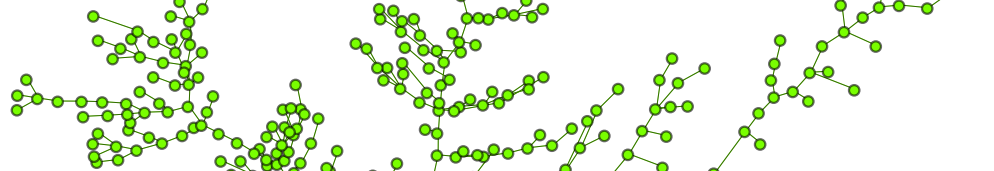

800000

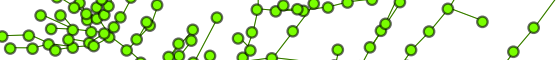

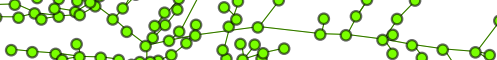

001000

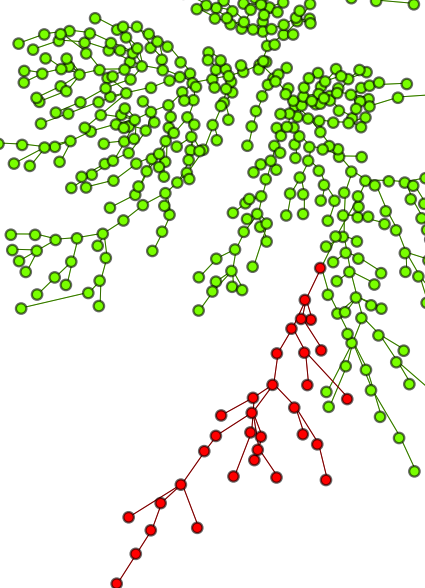

- NK cells

- non NK cells
B

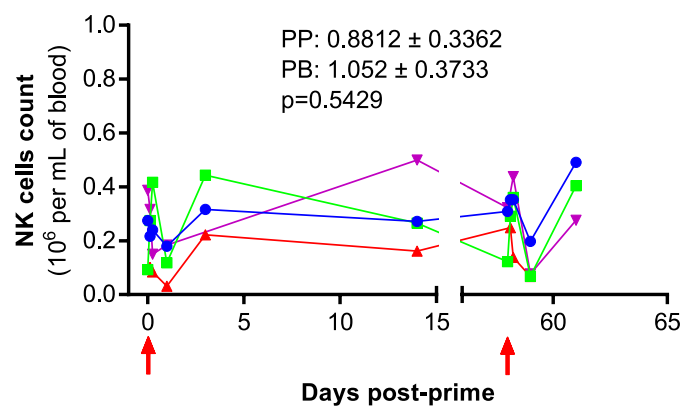

$\rightarrow$ BB078

- BB231

$\triangle \mathrm{BC} 641$

$\rightarrow$ BD620 

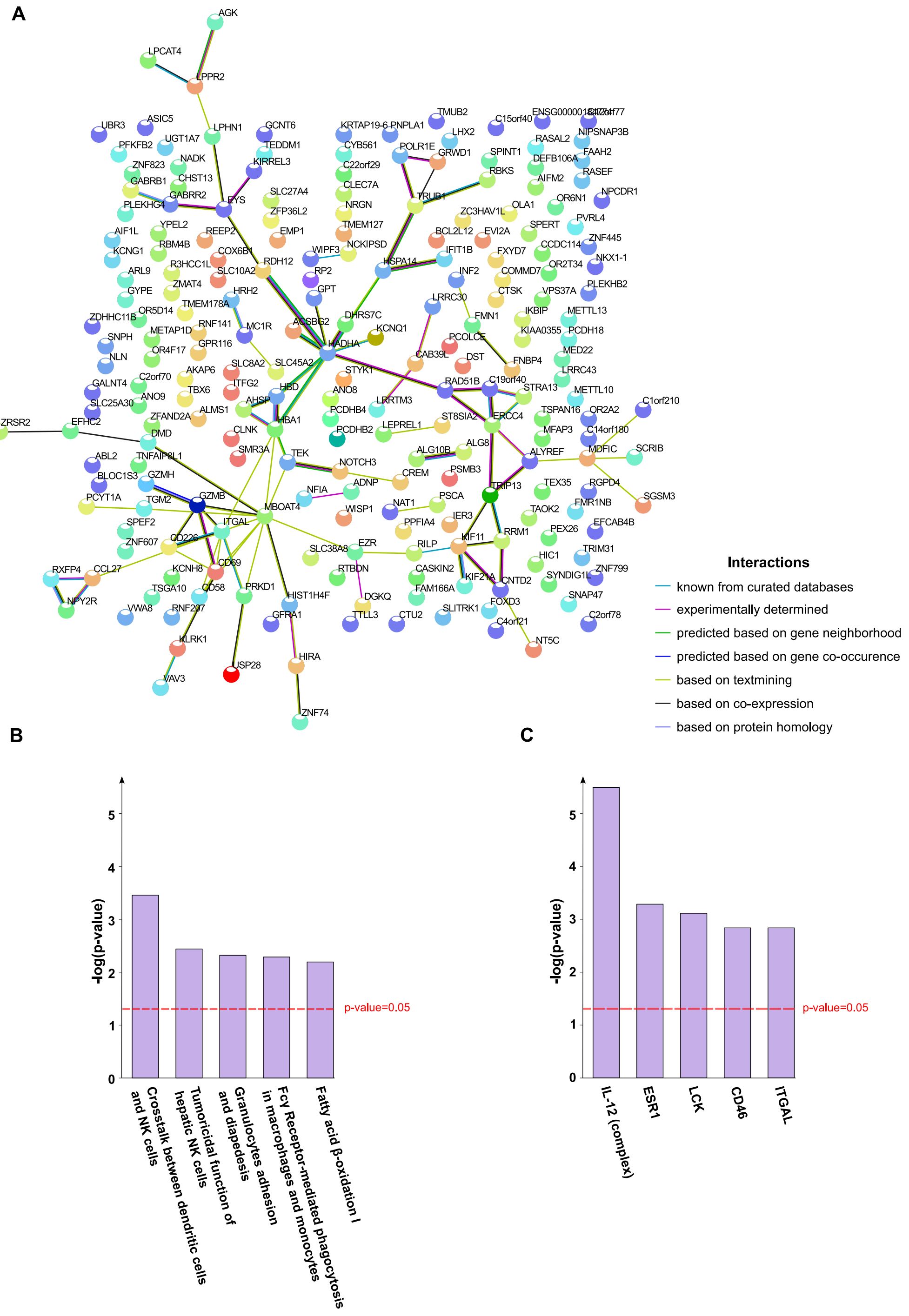


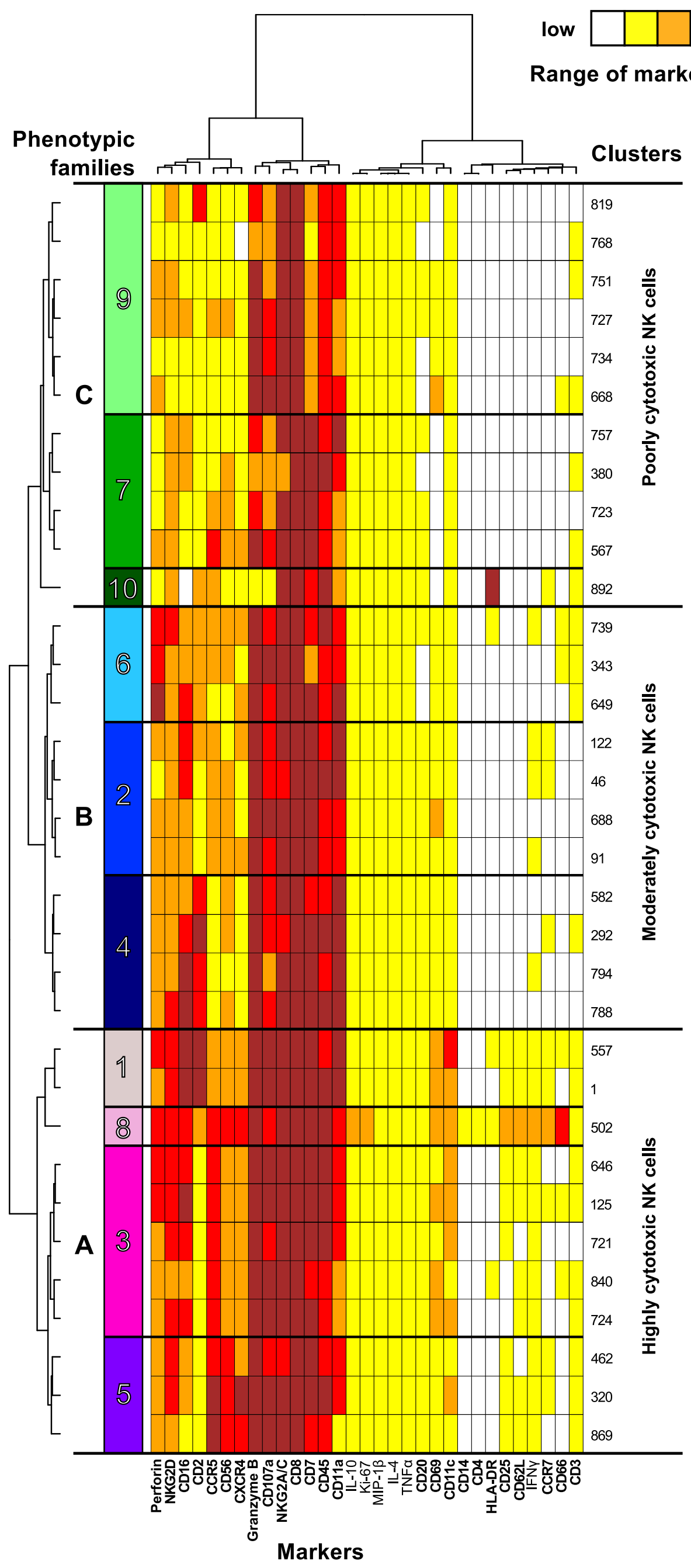



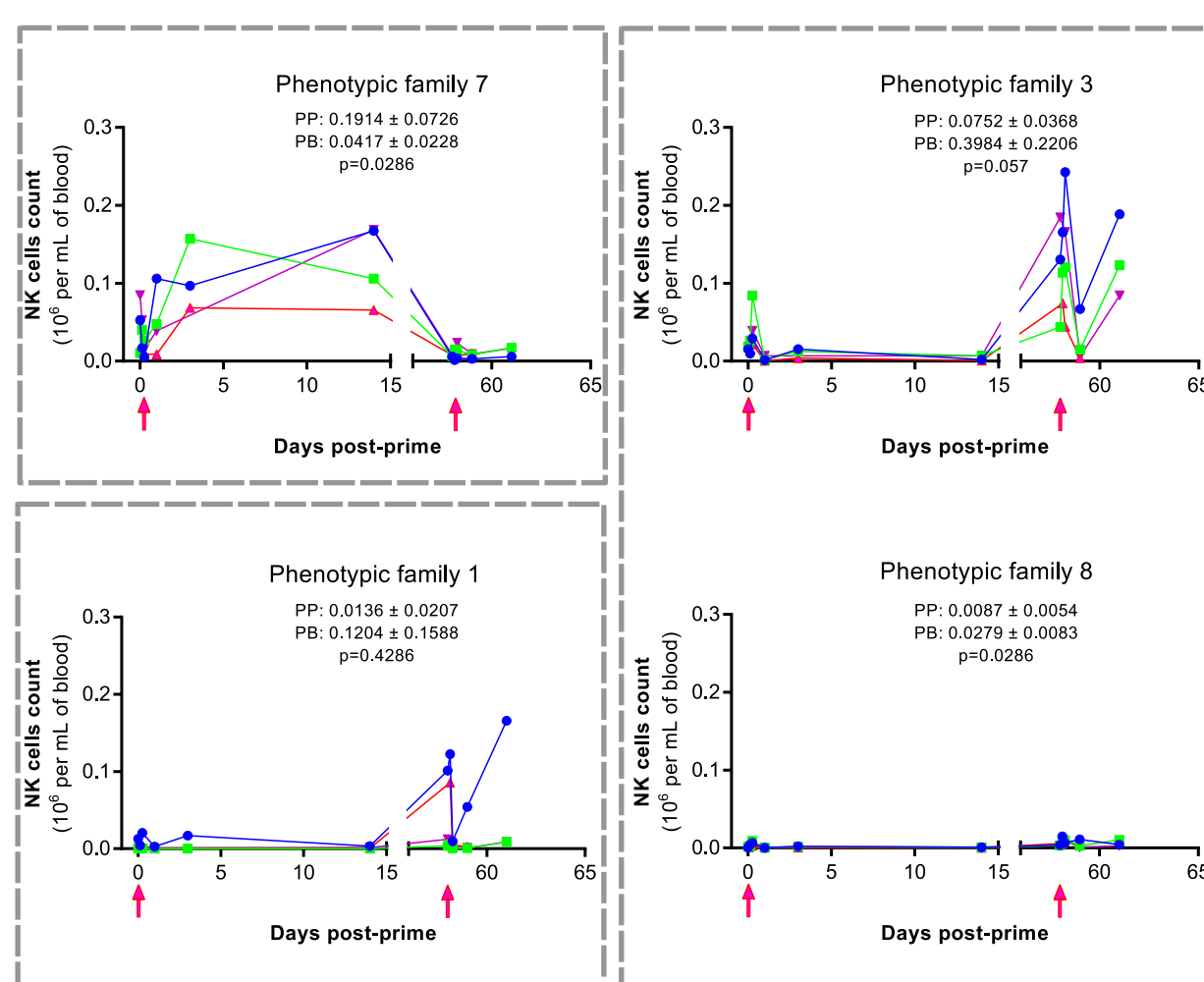

Phenotypic family 2

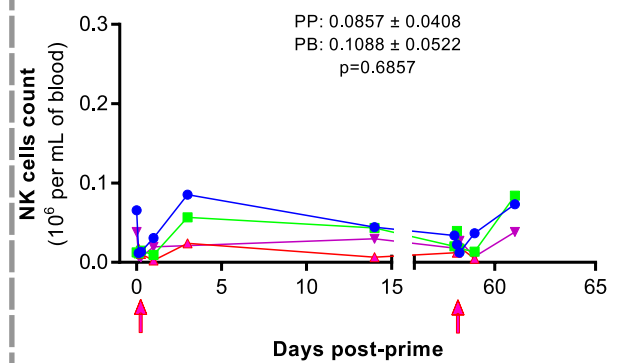

Phenotypic family 5

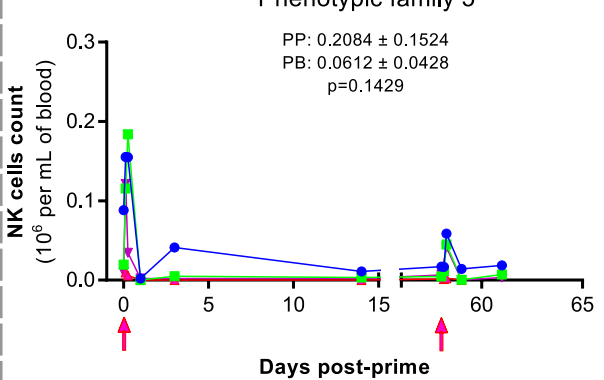

|

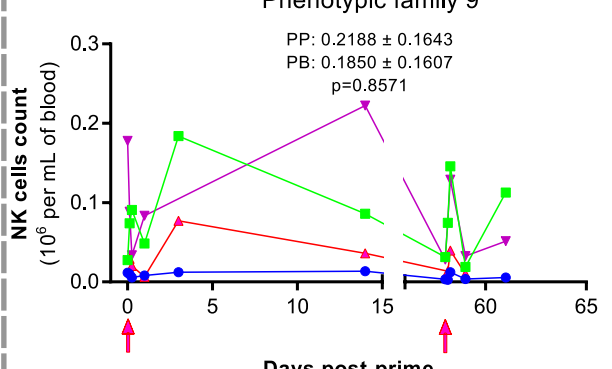

Days post-prime
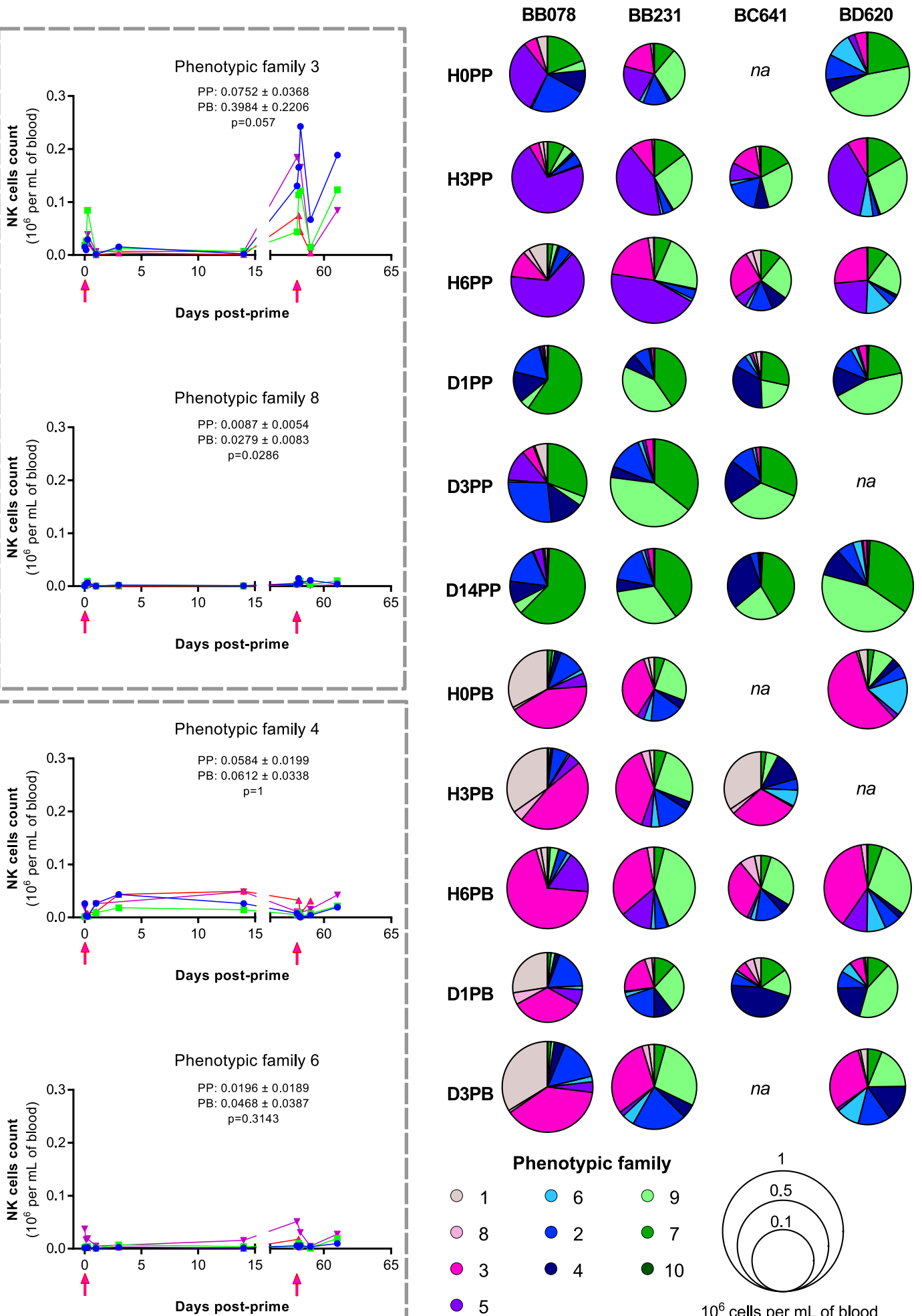

Phenotypic family

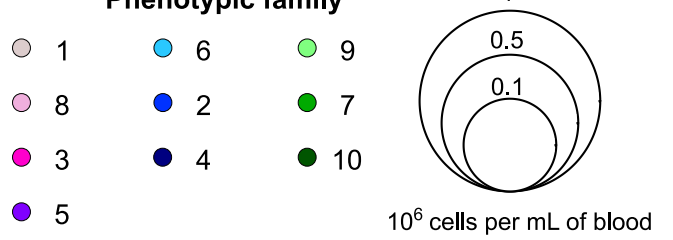

C

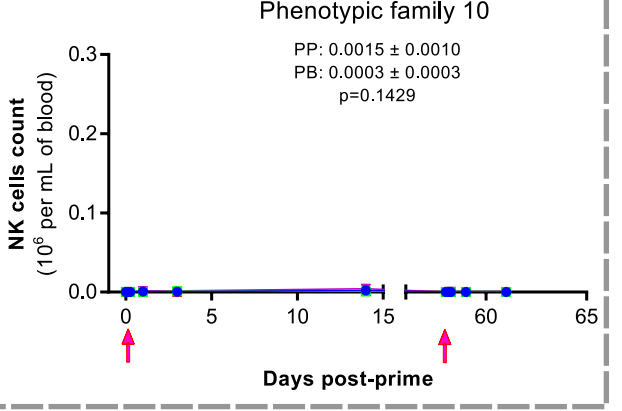

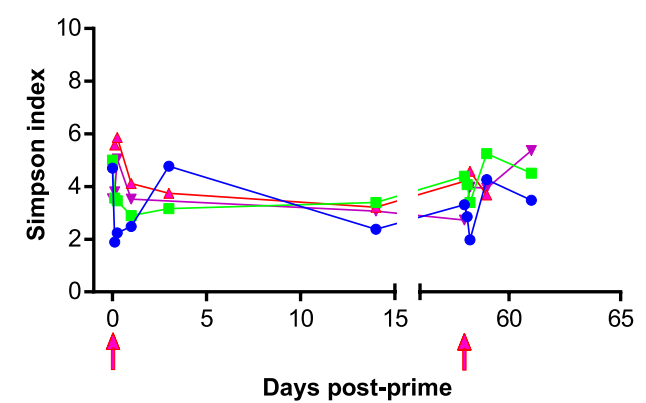

$$
\begin{aligned}
& -\quad B B 078 \\
& -\quad B B 231 \\
& -B C 641 \\
& -\quad B D 620
\end{aligned}
$$



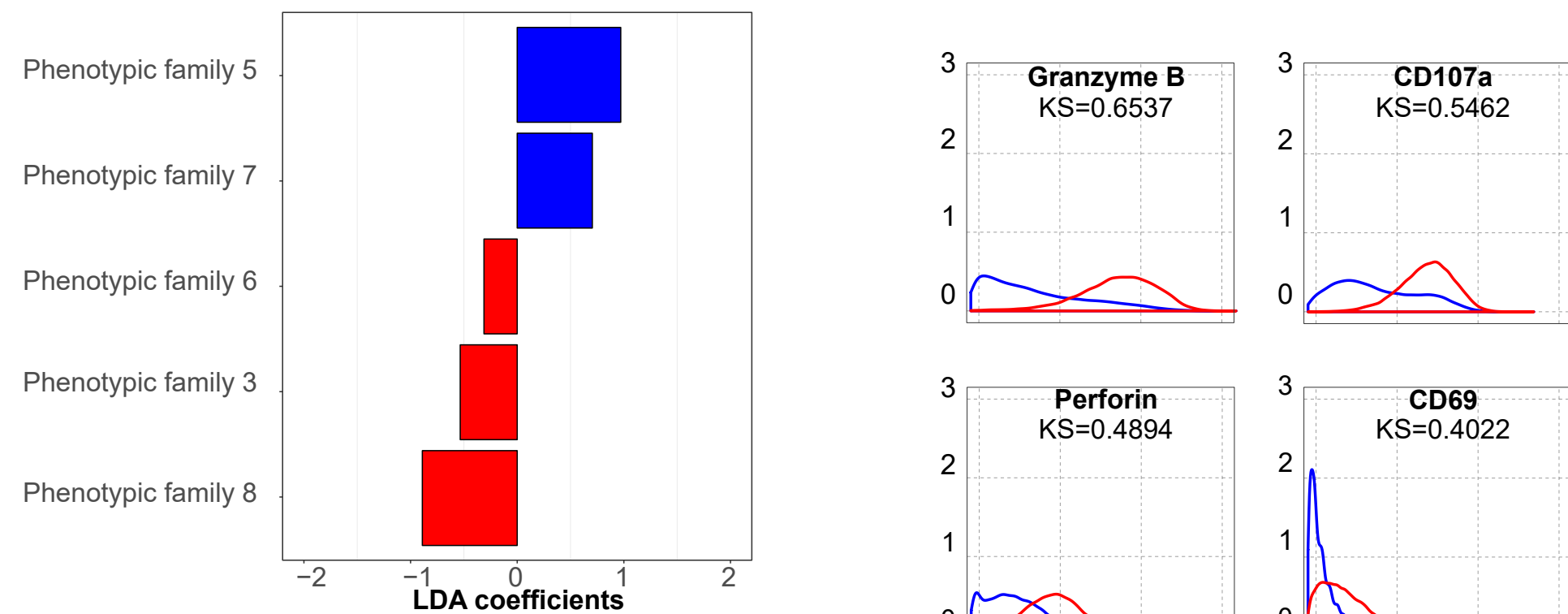

B

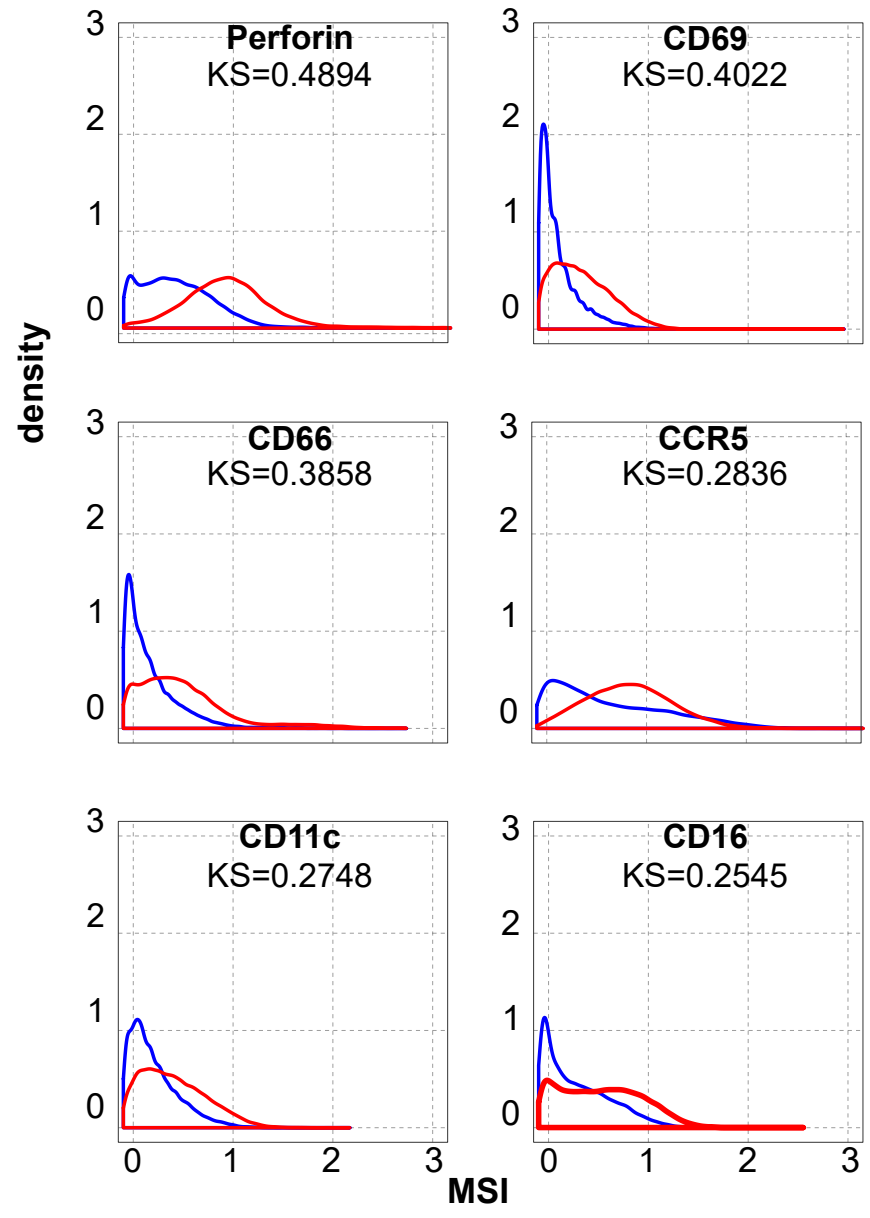

D
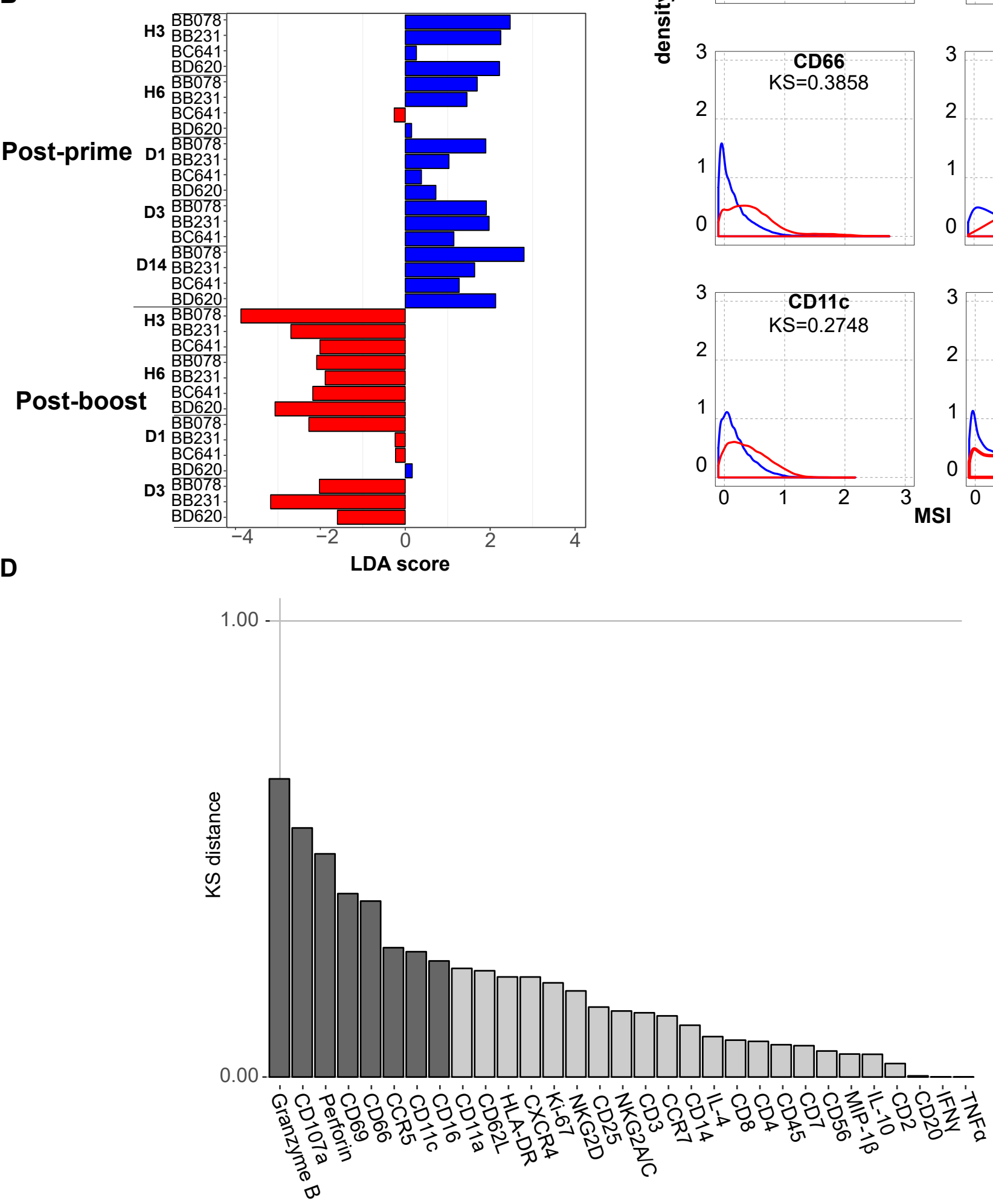


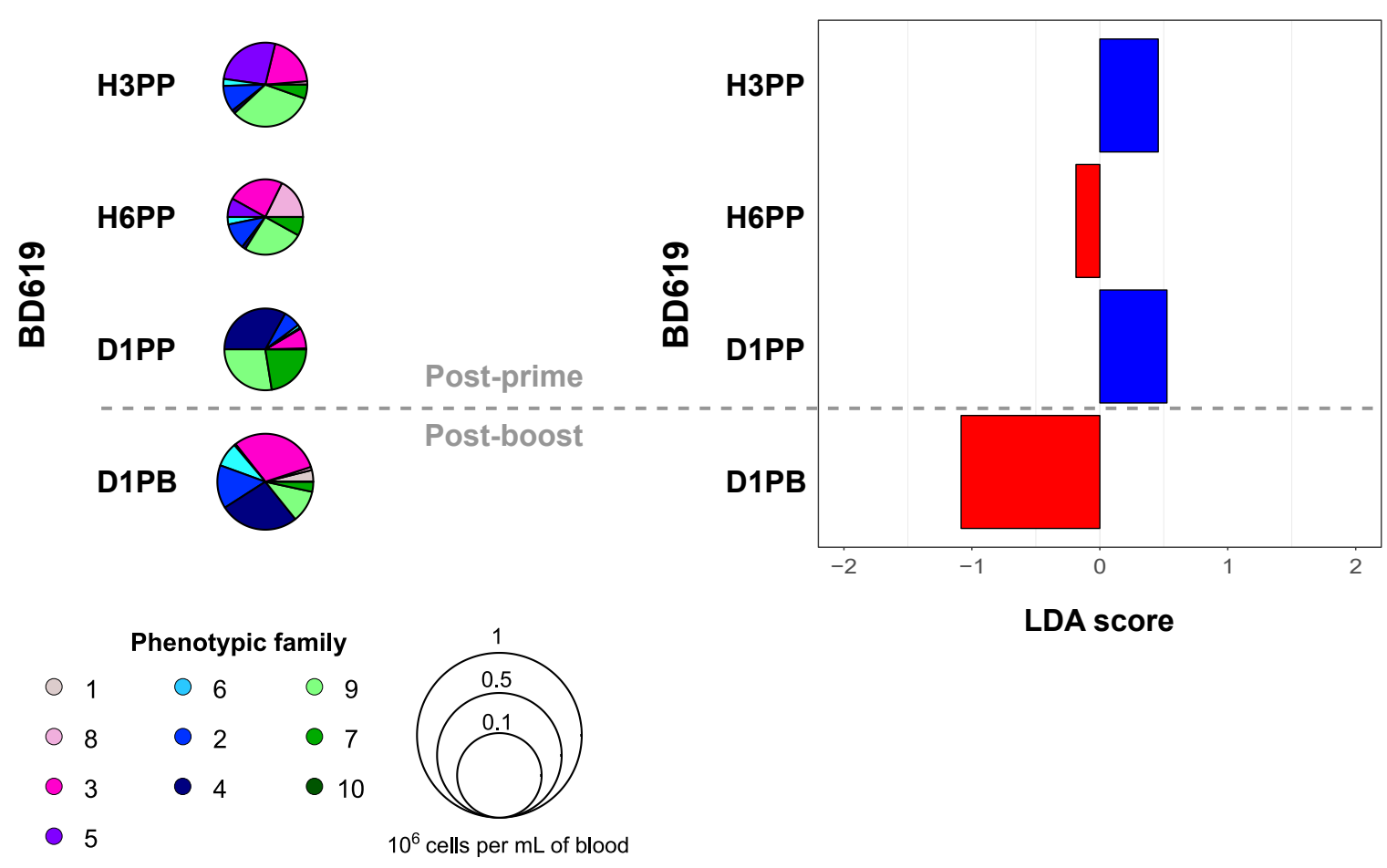


Highly cytotoxic CD56 ${ }^{\text {high }}$ CXCR4 $4^{\text {high }}$ Perforin ${ }^{\text {low }}$ CD $16^{\text {low }}$ Poorly cytotoxic NK cells

Highly cytotoxic CD56 $6^{\text {low }} \mathrm{CXCR} 4^{\text {low }}$ Perforin ${ }^{\text {high }} \mathrm{CD} 16^{\text {high }}$ Moderately cytotoxic NK cells

Poorly to highly cytotoxic NK cells

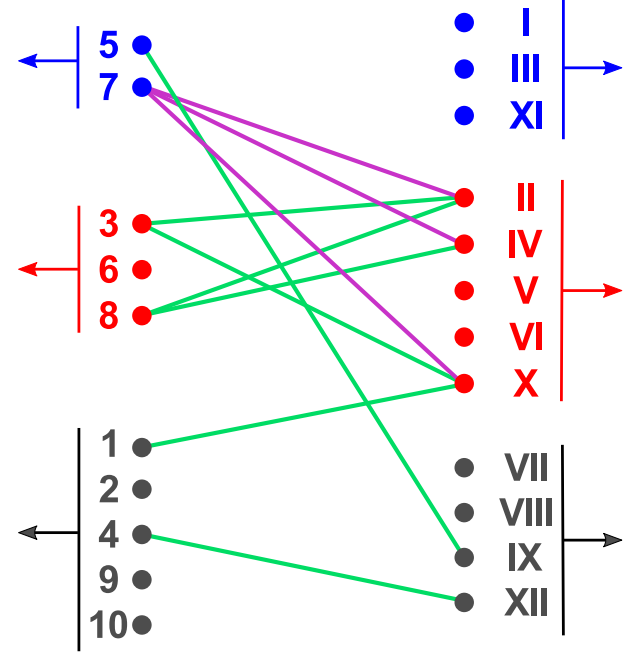

Basophils

Monocytes, including CD14 ${ }^{\text {low }}$ monocytes cDCs, including HLA-DR ${ }^{\text {low }}$ and $C D 64^{\text {high }} \mathrm{cDCs}$

Moderately/highly activated neutrophils Highly activated monocytes CCR5 ${ }^{\text {high }}$ CXCR $4^{\text {high }}$ cDCs inflammatory $\mathrm{cDCs}$ /non-classsical monocytes

eutrophils cD, including HLA-DR

\author{
prime signature \\ boost signature \\ not selected in LDA \\ positive correlation \\ negative correlation
}




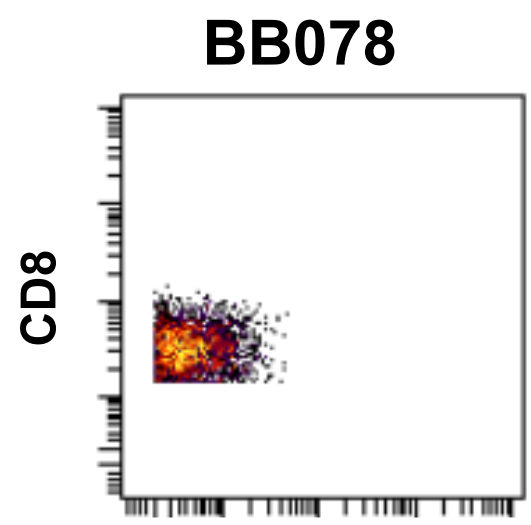

NKG2A/C

BC641

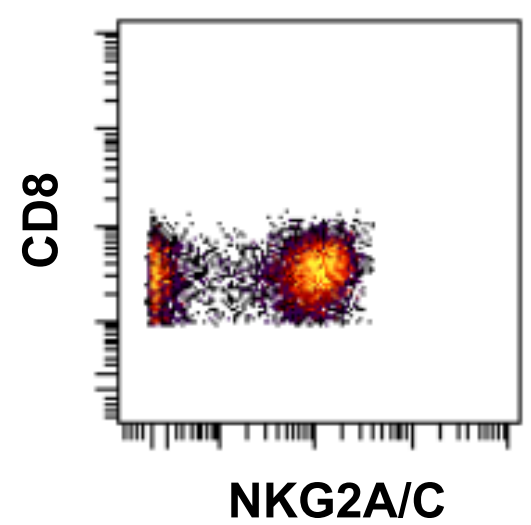

BB231

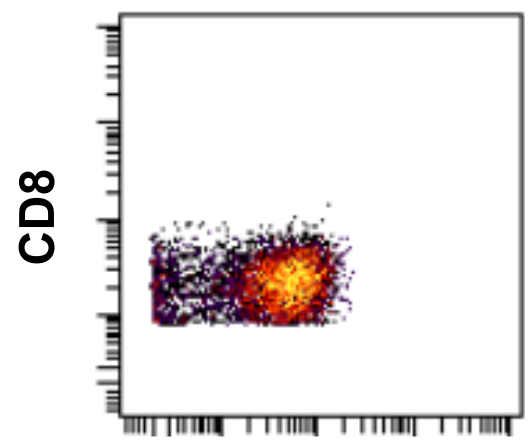

NKG2A/C

BD620

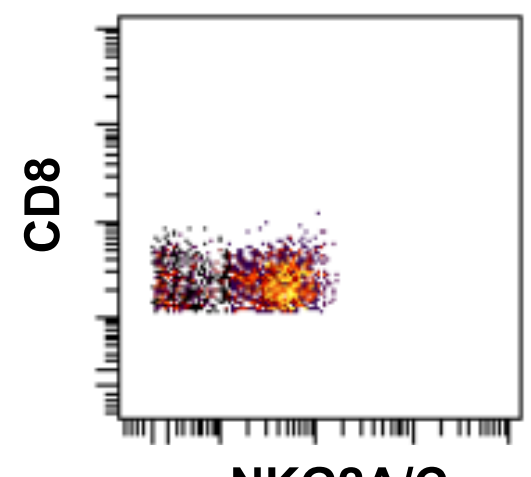

NKG2A/C

Figure S1. NKG2A/C inter-individual variability of expression. The biplot of CD8 vs. NKG2A/C is displayed for each animal at steady state (D19 before the prime). Macaque BB078 showed a different NKG2A/C staining pattern. 

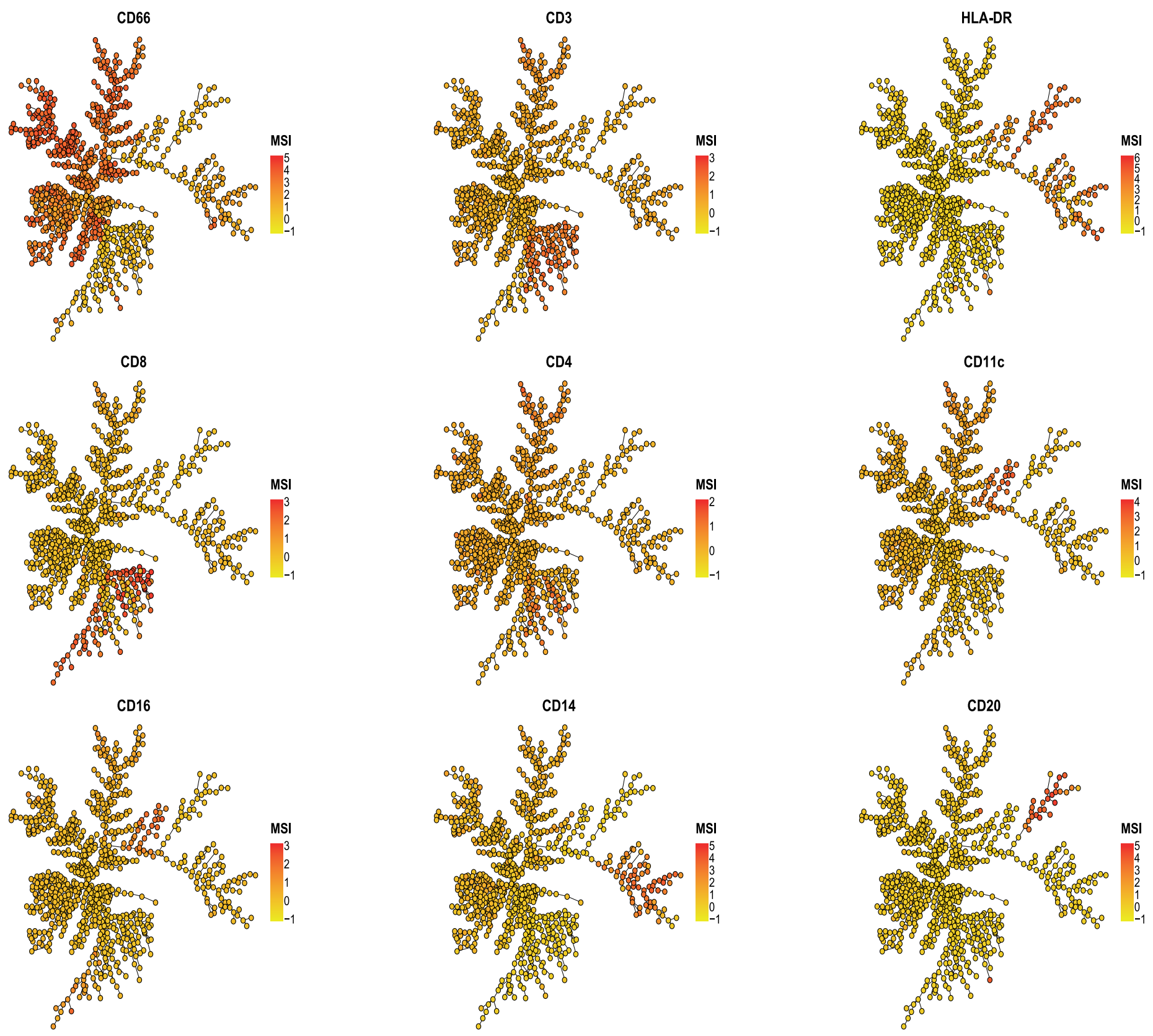

Figure S2. Annotation of the SPADE tree. Representation of the SPADE tree structure overlaid with the MSI for each indicated marker used to manually annotated the SPADE tree and identified NK cells as CD3- CD8+ cells. 
A

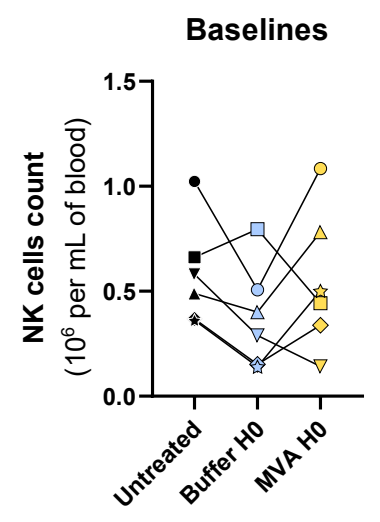

B

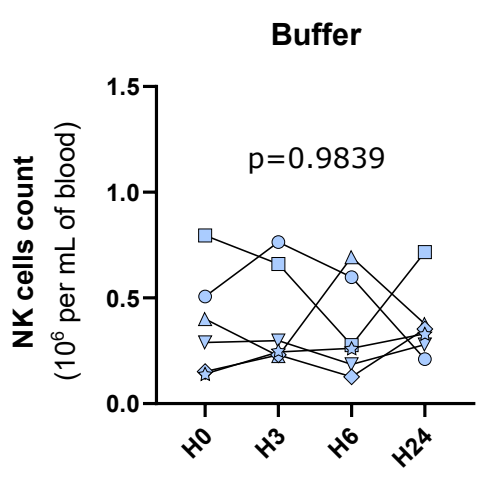

C

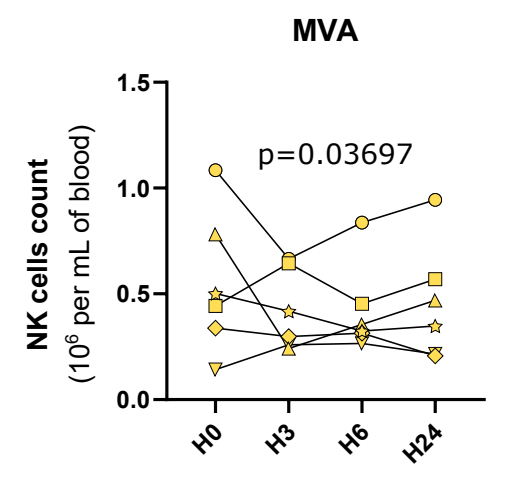

Figure S3. Specificity of vaccine-induced NK cell count decrease. (A) Variability of NK cell count across the three baselines. Dots from the same animal are linked together. (B) Evolution of NK cell count after buffer injection. (C) Evolution of NK cell number after MVA injection. (B-C) p-value: one-sample t-test on the changes of NK cell count compared to baseline for the three timepoints after immunization for all animals. (A-C) black: no injection, only anesthetic; blue: buffer; gold: MVA. 
Cluster 502

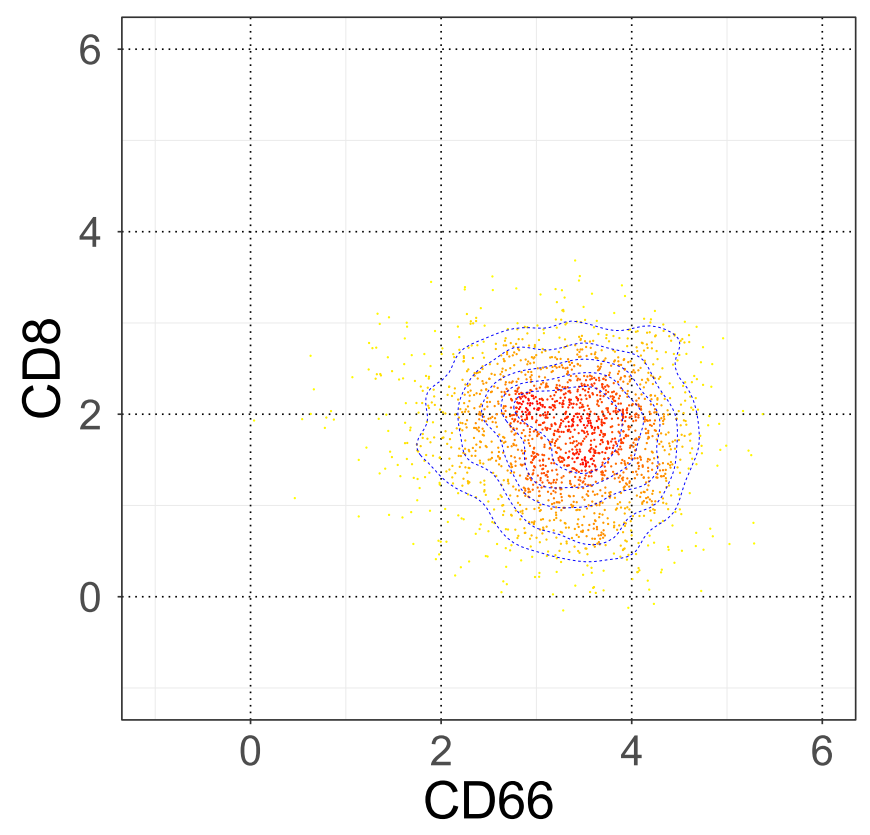

Cluster 892

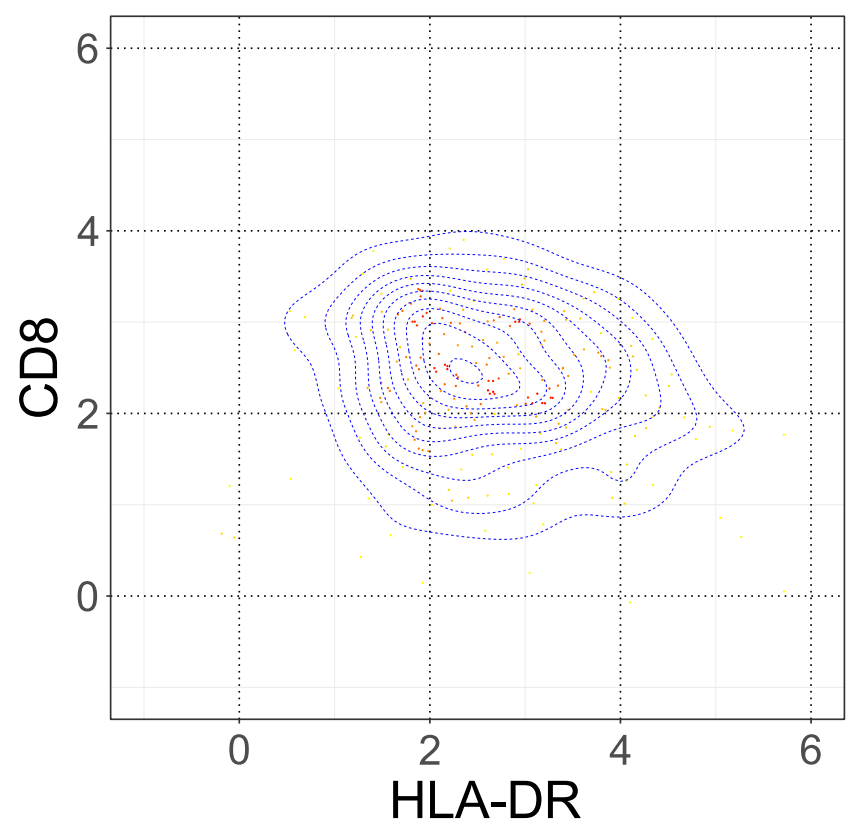

Figure S4. Peculiar NK cell clusters. Dotplots for NK cell clusters 502 and 892, each constituting its own phenotypic family, are displayed with the indicated markers, based on the merge of all samples (macaques and timepoints). They reveal the true coexpression of the indicated markers and not the co-existence of two cell population. Blue dotted line indicates density. 
A

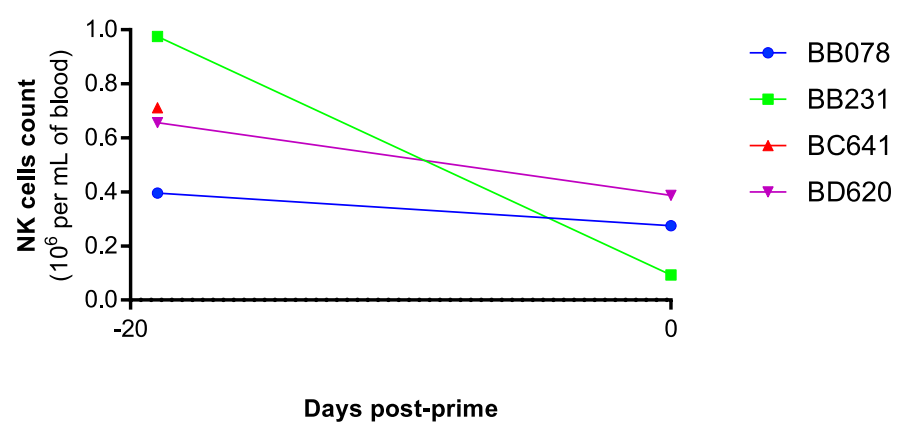

B

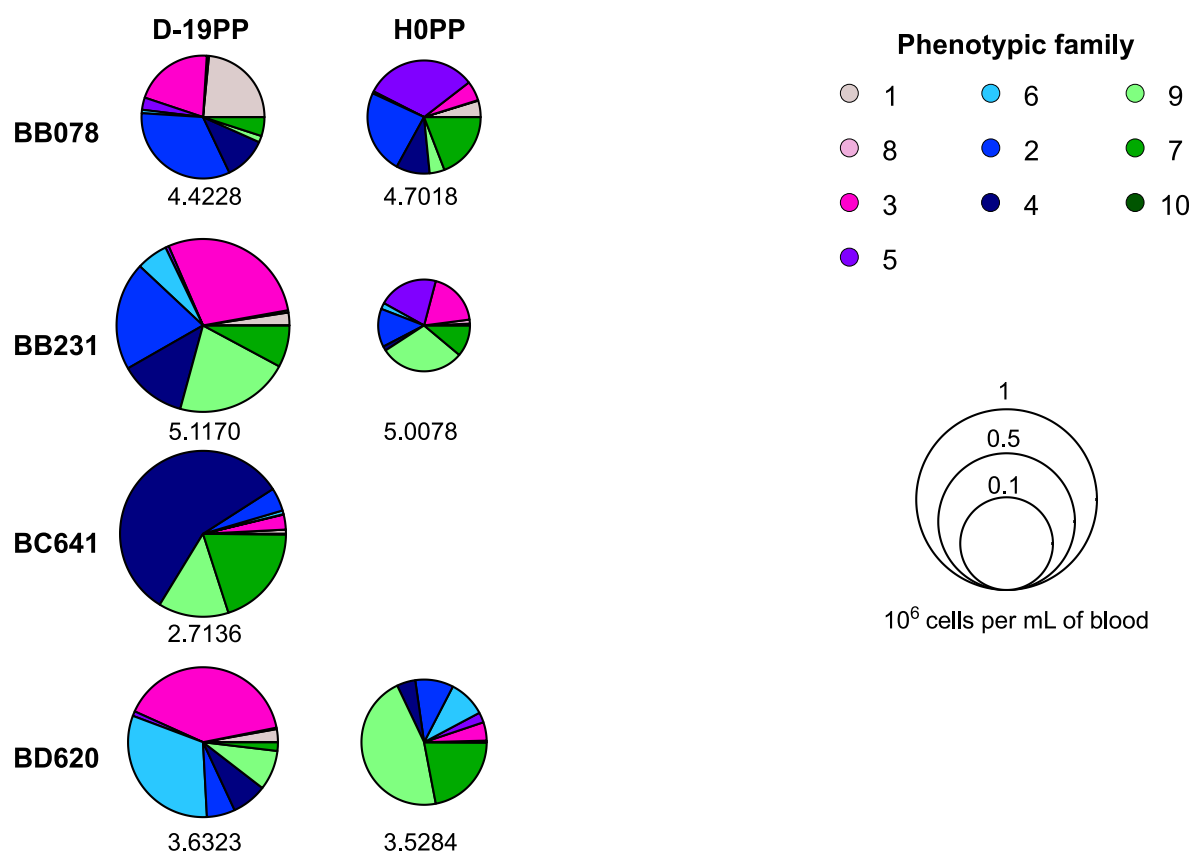

Figure S5. NK cells variability across baselines. (A) Absolute number of total NK cells is displayed for each of the two available baselines (D-19PP and HOPP) and for each animal. (B) The phenotypic family composition is given as well. The size of the pie is proportional to the absolute number of total NK cells. The inverse Simpson index, as a readout for diversity, is indicated below each pie. 
A

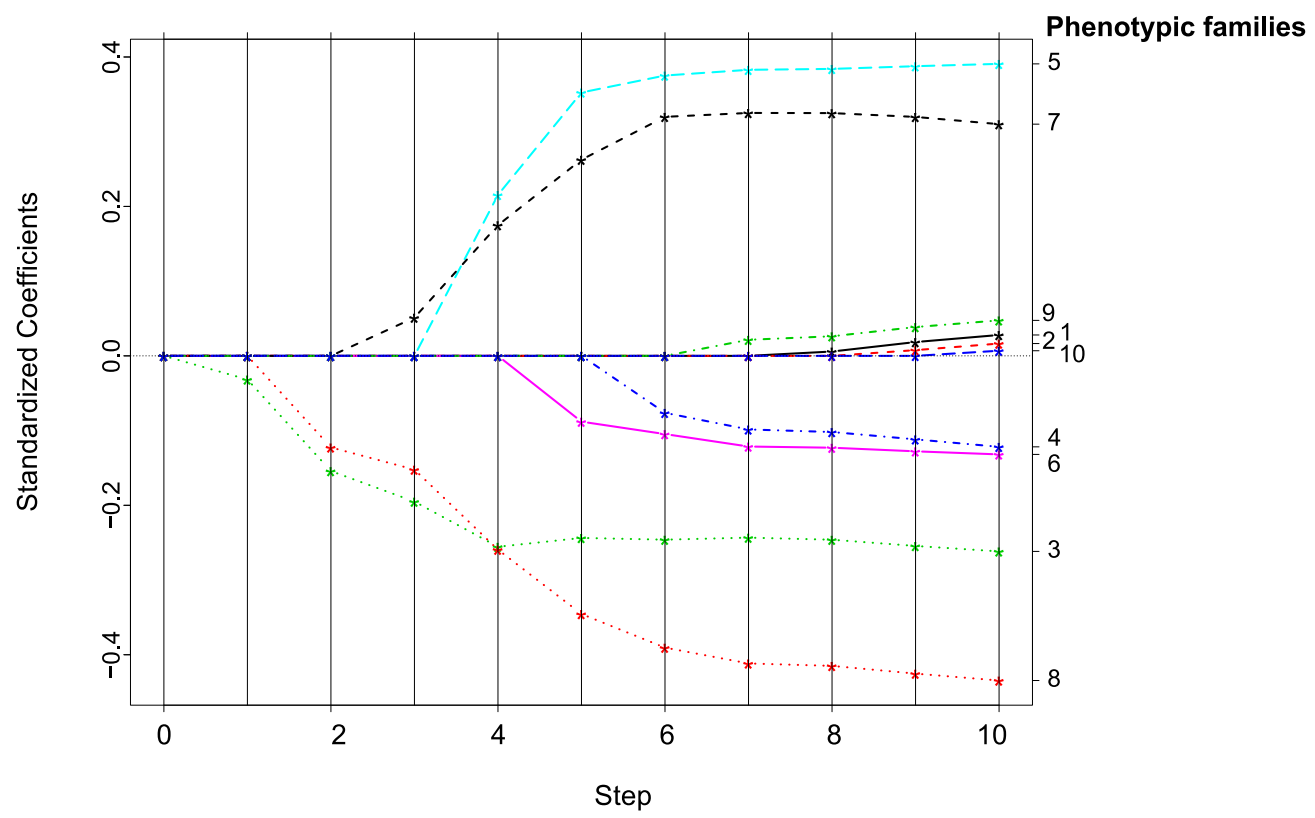

$\mathbf{B}$

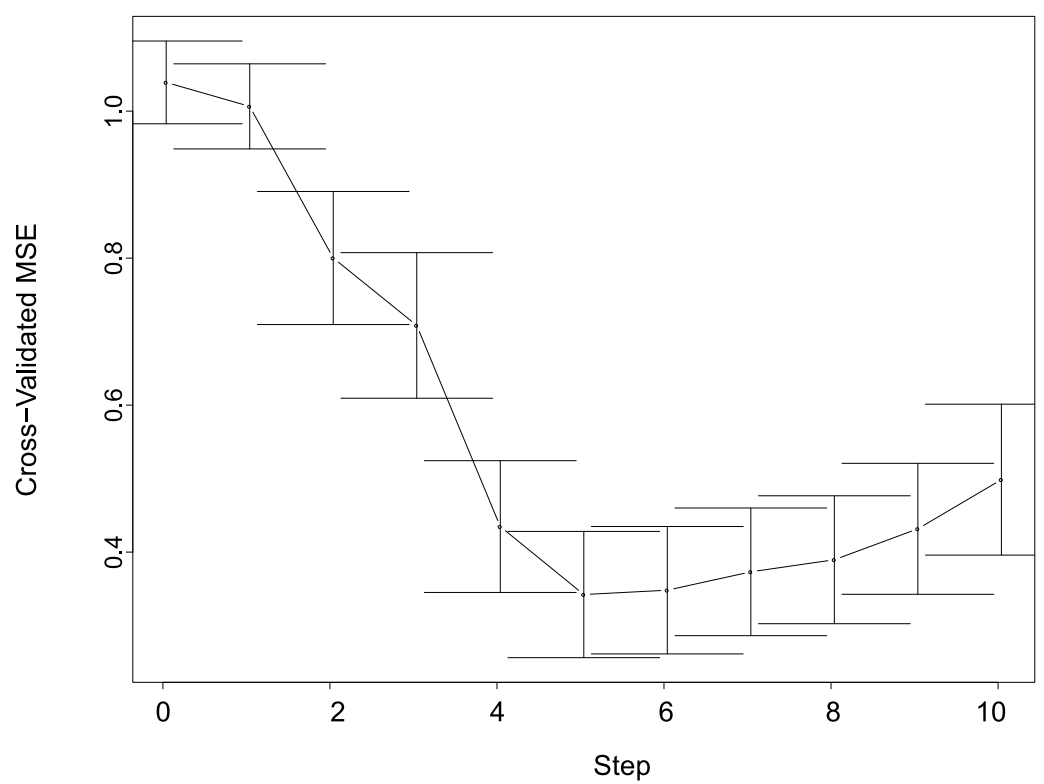

Figure S6. Selection of phenotypic families used for LDA classification using LASSO. (A) The evolution of LDA-LASSO coefficients at each step is represented for each phenotypic family. (B) The evolution of the mean standard error (MSE) at each step of the LASSO procedure, after leave-one-out cross-validation, is shown. 


\begin{tabular}{|c|c|c|c|c|}
\hline Metal & Marker & Clone & Surface & $\begin{array}{l}\text { Intra- } \\
\text { cellular }\end{array}$ \\
\hline $141 \mathrm{Pr}$ & CD66abce & TET2 & $\bullet$ & \\
\hline $142 \mathrm{Nd}$ & HLA-DR & L243 & $\bullet$ & \\
\hline $143 \mathrm{Nd}$ & CD3 & SP34.2 & $\bullet$ & \\
\hline $144 \mathrm{Nd}$ & CD107a & $\mathrm{H} 4 \mathrm{~A} 3$ & $\bullet$ & \\
\hline $145 \mathrm{Nd}$ & CD8 & RPAT8 & $\bullet$ & \\
\hline $146 \mathrm{Nd}$ & CD45 & D058-1283 & $\bullet$ & \\
\hline $147 \mathrm{Sm}$ & IL-4 & 7A3-3 & & $\bullet$ \\
\hline $148 \mathrm{Nd}$ & Granzyme B & GB11 & & $\bullet$ \\
\hline $149 \mathrm{Sm}$ & CD56 & NCAM16.2 & $\bullet$ & \\
\hline $150 \mathrm{Nd}$ & CD62L & SK11 & $\bullet$ & \\
\hline $152 \mathrm{Sm}$ & CD4 & L200 & $\bullet$ & \\
\hline 153Eu & CD11a & $\mathrm{HI} 111$ & $\bullet$ & \\
\hline $154 \mathrm{Sm}$ & CD2 & RPA2.10 & $\bullet$ & \\
\hline $155 \mathrm{Gd}$ & CD7 & M-T701 & $\bullet$ & \\
\hline $156 \mathrm{Gd}$ & MIP-1 $\beta$ & D21-1351 & & $\bullet$ \\
\hline 159Tb & TNF $\alpha$ & MAb11 & & $\bullet$ \\
\hline $160 \mathrm{Gd}$ & Ki-67 & B56 & & $\bullet$ \\
\hline 161Dy & NKG2D & $1 \mathrm{D} 11$ & $\bullet$ & \\
\hline 162Dy & CD11c & 3.9 & $\bullet$ & \\
\hline 164Dy & CD69 & FN50 & $\bullet$ & \\
\hline $165 \mathrm{Ho}$ & $\mathrm{IFNp}$ & B27 & & $\bullet$ \\
\hline $166 \mathrm{Er}$ & CD25 & 4 e 3 & $\bullet$ & \\
\hline $167 \mathrm{Er}$ & CD16 & $3 G 8$ & $\bullet$ & \\
\hline $168 \mathrm{Er}$ & CCR5 & $3 A 9$ & $\bullet$ & \\
\hline 169Tm & CXCR4 & $12 \mathrm{G} 5$ & $\bullet$ & \\
\hline $170 \mathrm{Er}$ & CD14 & M5E2 & $\bullet$ & \\
\hline $171 \mathrm{Yb}$ & Perforin & Pf-344 & & $\bullet$ \\
\hline $172 \mathrm{Yb}$ & $\mathrm{NKG} 2 \mathrm{~A} / \mathrm{C}^{\mathrm{a}}$ & Z199 & $\bullet$ & \\
\hline $174 \mathrm{Yb}$ & CD20 & $2 \mathrm{H} 7$ & $\bullet$ & \\
\hline 175Lu & CCR7 & G043H7 & $\bullet$ & \\
\hline 176Lu & IL-10 & JES3-9D7 & & $\bullet$ \\
\hline
\end{tabular}

Table 1. Antibody panel. Targeted markers, clones, and metals are shown. The right columns

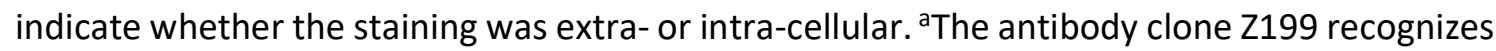
both NKG2A and NKG2C. 


\begin{tabular}{|c|c|c|c|c|}
\cline { 2 - 5 } \multicolumn{1}{c|}{} & BB078 & BB231 & BC641 & BD620 \\
\hline D-19PP & 76,557 & 60,206 & 60,143 & 52,082 \\
H0PP & 74,607 & 93,135 & & 67,358 \\
H3PP & 128,251 & 109,159 & 91,420 & 72,772 \\
H6PP & 124,104 & 161,898 & 128,497 & 66,482 \\
D1PP & 117,081 & 154,166 & 110,928 & 103,526 \\
D3PP & 79,789 & 81,863 & 76,958 & \\
D14PP & 116,972 & 108,706 & 124,386 & 86,044 \\
HOPB & 135,810 & 140,496 & & 92,991 \\
H3PB & 179,476 & 208,479 & 61,579 & \\
H6PB & 177,204 & 222,968 & 196,870 & 118,859 \\
D1PB & 257,189 & 167,840 & 243,434 & 116,967 \\
D3PB & 72,879 & 130,925 & & 95,656 \\
\hline
\end{tabular}

Table 2. Cells acquired with the CyTOF. For each sample, the number of leukocytes detected by the CyTOF (after exclusion of double positive $\mathrm{CD}^{+} \mathrm{CD}^{+} 6^{+}$eosinophils) is indicated. Not available samples are in grey. 


\begin{tabular}{|c|c|c|c|}
\hline Markers & $\begin{array}{c}\text { Number of } \\
\text { non-uniform } \\
\text { NK cell clusters }\end{array}$ & $\begin{array}{c}\text { Percentage of } \\
\text { non-uniform } \\
\text { NK cell clusters }\end{array}$ & $\begin{array}{c}\text { ID of non-uniform } \\
\text { NK cell clusters }\end{array}$ \\
\hline CD2 & 4 & 12 & $582,739,788,892$ \\
CD16 & 4 & 12 & $122,380,721,788$ \\
Perforin & 3 & 9 & $723,757,819$ \\
CD4 & 1 & 3 & 582 \\
CD7 & 1 & 3 & 567 \\
HLA-DR & 1 & 3 & 567 \\
NKG2A/C & 1 & 3 & 892 \\
CCR5 & 0 & 0 & - \\
CCR7 & 0 & 0 & - \\
CD3 & 0 & 0 & - \\
CD8 & 0 & 0 & - \\
CD11a & 0 & 0 & - \\
CD11c & 0 & 0 & - \\
CD14 & 0 & 0 & - \\
CD20 & 0 & 0 & - \\
CD25 & 0 & 0 & - \\
CD45 & 0 & 0 & - \\
CD66abce & 0 & 0 & - \\
CD62L & 0 & 0 & - \\
CD69 & 0 & 0 & - \\
CD107a & 0 & 0 & - \\
CXCR4 & 0 & 0 & - \\
Granzyme B & 0 & 0 & - \\
NKG2D & 0 & 0 & - \\
\hline
\end{tabular}

Table 3. Number and percentage of non-uniform NK cell clusters. The number, percentage, and ID of NK cell clusters that do not reach the condition of uniformity are shown. 